\title{
Chelation and Stereodirecting Group Effects on Regio- and Diastereoselective Samarium(II)-Water Allylic Benzoate Reductions
}

\author{
Trevor F. Stockdale \\ Michael A. Leitch \\ Gregory W. O'Neil*
}

Department of Chemistry, Western Washington University, 516 High Street, Bellingham, WA 98225, USA

oneilg@wwu.edu

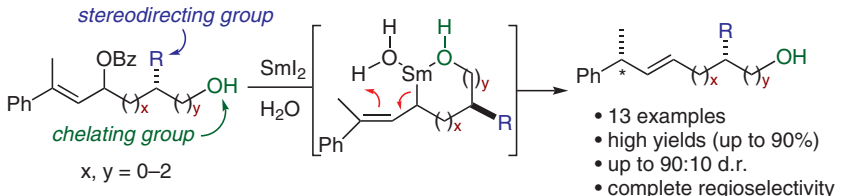

Our group became interested in the use of $\mathrm{SmI}_{2}$ for the reduction of acyloxysulfones as part of a masked-alkene metathesis protocol. ${ }^{11}$ More recently we reported that allylic benzoates $1 \mathbf{a}$ or $\mathbf{1 b}$ can be reduced with $\mathrm{SmI}_{2}$ in the presence of an alcohol additive $\left(\mathrm{R}^{\prime} \mathrm{OH}\right){ }^{12}$ converging to the corresponding reduced products 2 with high regioselectivity (Scheme 1). The regioselectivity of this reaction can be rationalized by steric considerations of the organosamarium intermediate, and a pericyclic protonation mechanism involving a samarium-bound alcohol molecule. This method then featured in our synthesis of the biologically active natural product honokiol to simultaneously install both allyl substituents found in the target compound from the bisallylic benzoate precursor $3 .^{13}$

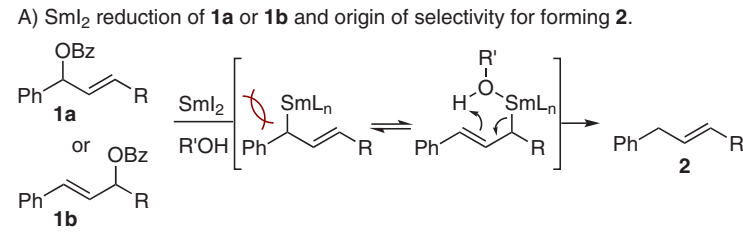

B) Application of $\mathrm{Sml}_{2}$ allylic benzoate reduction to the synthesis of honokiol.

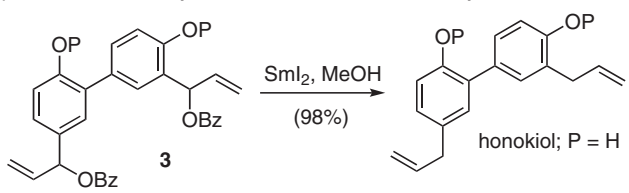

Scheme 1 (A) Regioselective $\mathrm{Sml}_{2}$ allyl benzoate reductions and (B) its application to a synthesis of honokiol

Applied to trisubstituted alkene-containing substrates, we recognized that the reaction would generate a new stereocenter $\left({ }^{*}\right)$, and became interested in finding ways to develop this reaction as a new strategy for stereoselective synthesis (Scheme 2). ${ }^{14}$ We hypothesized that incorporation of a Lewis basic chelating element (e.g., OP) and stereo- 
directing group $(\mathrm{R})$ would render $\mathrm{SmI}_{2}\left(\mathrm{H}_{2} \mathrm{O}\right)_{n}$ reductions of compounds of type 4 both regio- and diastereoselective. Together, these groups could impart facial selectivity during the intramolecular protonation event from a chelated intermediate. The importance of chelation to our design was supported by the reduction of compound $\mathbf{5}$ to product $\mathbf{6}$, which proved to be non-diastereoselective.

$$
\text { B) }
$$

Scheme 2 (A) Proposed regio- and diastereoselective allylic benzoate reductions proceeding through a chelated organosamarium intermediate. (B) The importance of chelation for stereoselectivity is supported by the non-diastereoselective reaction of compound $\mathbf{5}$, which lacks this ability.

For the chelating element we chose to focus on oxygen given the well-established oxophilicity of samarium. ${ }^{15}$ This also introduced an obvious synthetic disconnection (i.e., a carbonyl addition) when designing the synthesis of our desired substrates. With these considerations in mind, initial investigations began with the preparation of compound $\mathbf{8}$ by zirconium-catalyzed carboalumination ${ }^{16}$ of phenyacetylene and addition of the resulting vinylalane into Roche ester-derived aldehyde $(S)-7^{17}$ (Scheme 3 ). The stereochemistry of the newly formed hydroxyl in $\mathbf{8}$ for the major isomer is assumed to be $(S)$ arising from chelation control, ${ }^{18}$ but was not rigorously determined as this stereocenter proved unimportant for the subsequent eliminations (vide infra). After benzoylation, unfortunately the reduction of $\mathbf{9}$ in the presence of various additives proceeded with low diastereoselectivity (50:50 to 60:40) and only modest regioselectivity (up to 5:1).

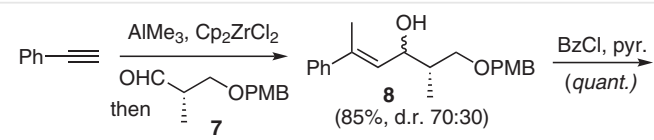

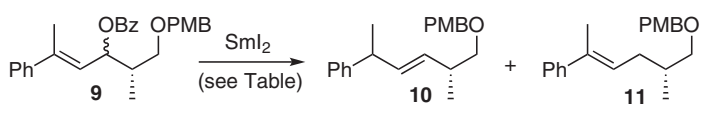

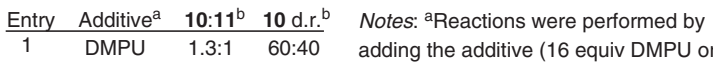

$$
\begin{aligned}
& 2 \quad t \text { - } \mathrm{BuOH} \quad 3: 1 \quad 69: 31 \quad 1400 \text { equiv } \mathrm{ROH} \text { ) to } \mathrm{Sml}_{2} \text { (7 equiv) } \\
& 3 \quad \mathrm{MeOH} \quad 3: 1 \quad 60: 40 \text { followed by the substrate at room } \\
& \begin{array}{llll}
4 & \mathrm{H}_{2} \mathrm{O} & 5: 1 & 50: 50 \\
\end{array}
\end{aligned}
$$

Scheme 3 Synthesis of substrate 9 and initial screening of its reduction with $\mathrm{Sml}_{2}\left(\mathrm{H}_{2} \mathrm{O}\right)_{n}$ in the presence of various additives

Gratifyingly after PMB-removal, reduction of the corresponding free hydroxyl compound 12 proceeded with both enhanced regio- and diastereoselectivity (d.r.), presumably as a result of greater chelation to samarium, ${ }^{19}$ although secondary coordination sphere effects (e.g., hydrogen-bond networks) cannot be ruled out at this time (Scheme 4, Table 1). ${ }^{8,10 a}$ The impact of different additives on the outcome of the reaction with 12 were investigated as outlined in Table 1. Interestingly, the highest (and nearly identical) diastereoselectivities were obtained using either anhydrous conditions (DMPU; entry 1 ) followed by quenching $\left(\mathrm{aq} \mathrm{NH}_{4} \mathrm{Cl}\right.$ ) or in the presence of water (entry 5$),{ }^{20}$ suggestive against an internal protonation by the hydroxyl group per se (performing the reaction in $\mathrm{D}_{2} \mathrm{O}$ resulted in $>90 \%$ deuterium incorporation by ${ }^{1} \mathrm{H}$ NMR at $\mathrm{C} 5$ ). Both reactions produced compound 13 as a 3:1 mixture of diastereomers and exclusively as the trans-isomer, however, regioselectivity for the DMPU reaction was much lower ( $2: 1$ vs $15: 1$ for $\mathrm{H}_{2} \mathrm{O}$ ). Colder conditions (i.e., $0{ }^{\circ} \mathrm{C}$, entry 6 ) also led to an erosion of regioselectivity. The reaction proved non-stereospecific to the stereochemistry of the $\mathrm{OBz}$ stereocenter, with identical results obtained when 12 was used as a 50:50 (entry 7) or 70:30 (entry 5, ref. Scheme 3) mixture of diastereomers. ${ }^{21}$ This is attractive from a synthetic standpoint, allowing us to prepare and use substrates epimeric at this position without any impact on the subsequent reductions. The amount of water used also had little effect on the d.r. of the reactions [e.g., 76:24 for 70 equiv (entry 8) vs 75:25 for 1400 equiv (entry 6)], consistent with other studies showing that even high concentrations of water do not lead to complete saturation of Sm(II). ${ }^{3 \mathrm{~b}}$ Regioselectivity, however, tended to be higher at fewer equivalents of $\mathrm{H}_{2} \mathrm{O}$, perhaps as a result of a competing intermolecular protonation at the higher equivalents (ref. Scheme 1). Yields also increased with decreased $\mathrm{H}_{2} \mathrm{O}$ [with the exception of 1 equiv (66\% yield)], where side-products that we have tentatively assigned as radical dimers were observed. ${ }^{22}$ The absolute configuration of the newly formed stereocenter was determined by ozonolysis of 13 and comparison of the optical activity of the resulting aldehyde $\mathbf{1 5}$ to that previously reported. ${ }^{23}$ This analysis revealed that the sample was enriched in the $(S)$ $(+)$-enantiomer, indicating that the major diastereomer of 13 had the $(2 R, 5 R)$-configuration. Our working model to explain the stereochemical outcome of this reaction is based on the ring-conformation energetics of a fused 5,6-bicyclic organosamarium transition state structure Sm-I, ${ }^{24}$ involving hydroxyl chelation of samarium ${ }^{25}$ followed by intramolecular protonation by a coordinated water molecule.

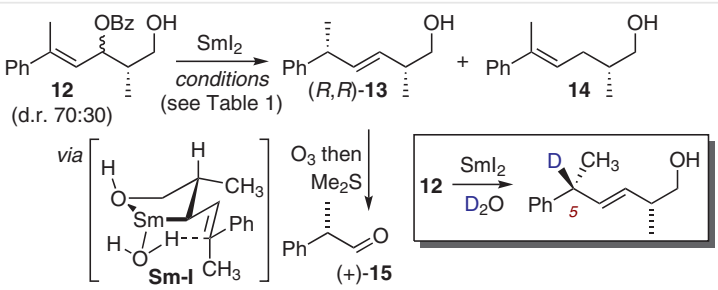

Scheme $4 \mathrm{Sml}_{2}$ reduction of compound 12 and determination of absolute stereochemistry by ozonolysis of $\mathbf{1 3}$ 
Table 1 Additive Effects on $\mathrm{Sml}_{2}$ Reductions of Compound 12 (ref. Scheme 4)

\begin{tabular}{lccc}
\hline Entry & Additive $^{\text {a }}$ & $\mathbf{1 3 : 1 4 ^ { b }}$ & d.r. ${ }^{\text {b }}$ of $\mathbf{1 3}$ \\
\hline 1 & DMPU & $2: 1$ & $75: 25$ \\
2 & $t-\mathrm{BuOH}$ & $1: 0^{c}$ & $67: 33$ \\
3 & $i-\mathrm{PrOH}$ & $2.3: 1$ & $67: 33$ \\
4 & $\mathrm{MeOH}$ & $1: 0^{c}$ & $60: 40$ \\
5 & $\mathrm{H}_{2} \mathrm{O}$ & $15: 1$ & $76: 24$ \\
6 & $\mathrm{H}_{2} \mathrm{O}^{\text {d }}$ & $5: 1$ & $75: 25$ \\
7 & $\mathrm{H}_{2} \mathrm{O}^{\mathrm{e}}$ & $15: 1$ & $76: 24$ \\
8 & $\mathrm{H}_{2} \mathrm{O}^{\mathrm{f}}$ & $15: 1$ & $76: 24$ \\
\hline
\end{tabular}

a Reactions were performed by adding the additive (16 equiv DMPU or 1400 equiv $\mathrm{ROH}$ ) to $\mathrm{Sml}_{2}$ (7 equiv) followed by the substrate and stirring for $30 \mathrm{~min}$.

${ }^{\mathrm{b}}$ Determined by ${ }^{1} \mathrm{H}$ NMR spectroscopy.

'Compound 14 was not detected by NMR spectroscopy.

d Performed at $0^{\circ} \mathrm{C}$.

e Compound 12 was used as a 1:1 mixture of diastereomers.

${ }^{\mathrm{f}}$ Performed using 70 equiv of $\mathrm{H}_{2} \mathrm{O}$.

In thinking about other suitable and available aldehyde starting materials from which we could prepare additional substrates to further investigate this transformation, we were drawn to lactate-derived aldehyde $\mathbf{1 6}^{26}$ (Scheme 5). Using similar chemistry to that employed in the synthesis of 12, we prepared compounds 19 and $\mathbf{2 0}$ and investigated their reduction with $\mathrm{SmI}_{2}\left(\mathrm{H}_{2} \mathrm{O}\right)_{\mathrm{n}}$. Treatment of $\mathbf{1 9}$ or $\mathbf{2 0}$ to our optimized conditions from experiments with 12 (e.g., 15 equiv $\mathrm{H}_{2} \mathrm{O}$ relative to $\mathrm{SmI}_{2}$, r.t.) gave the desired products $\mathbf{2 1}$ or $\mathbf{2 2}$ with complete regioselectivity and high diastereoselectivity [84:16; higher than for compound 12 (75:25)], however, in low yield due to a competing elimination and formation of the corresponding diene (presumably $\beta$-elimination of the hydroxyl group after benzoate cleavage). Increasing the equivalents of water to either 100 or 200 equivalents suppressed this elimination to some extent (presumably by increasing the rate of protonation), allowing for the isolation of $\mathbf{2 1}$ in $60 \%$ yield. The highest d.r. (90:10) was obtained for the $n$-butyl substrate $\mathbf{2 0}$ using 50 equivalents of water, giving compound $\mathbf{2 2}$ in $50 \%$ isolated yield. The absolute configuration of the newly formed stereocenter was determined by ozonolysis of the product which produced primarily $(S)-(+)$-aldehyde 15 by polarimetry. ${ }^{23}$ A possible model to explain this selectivity based on that previously proposed for the one-carbon homologated samarium intermediate is shown in Scheme 5, with the organosamarium transition state structure $\mathbf{S m}$-II existing in this case as an $\eta^{3}$-complex. ${ }^{27}$

Comparing results for lactate substrate $\mathbf{1 9}$ and Roche ester-derived compound $\mathbf{1 2}$ demonstrates that the location of the hydroxyl group (i.e., linker length) can impact both the yield and selectivity for this reaction. To further investigate this effect, we synthesized and examined the $\operatorname{SmI}_{2}\left(\mathrm{H}_{2} \mathrm{O}\right)_{n}$ reductions of compounds 25 and $\mathbf{3 0}$, which contain hydrox-

$$
\begin{aligned}
& \mathrm{R}=\frac{\mathrm{AlMe}_{3}, \mathrm{Cp}_{2} \mathrm{ZrCl}_{2}}{\mathrm{OHC} \bigcup_{\vdots}^{\mathrm{OPMB}}} \stackrel{\mathrm{R}}{\longrightarrow} \underbrace{\mathrm{O}_{0}}_{\vdots} \mathrm{OPMB} \frac{1 . \mathrm{BzCl}, \mathrm{pyr} .}{2 . \mathrm{DDQ}} \\
& \text { then } \leqq 16 \quad \mathrm{R}=\mathrm{Ph}, 17,82 \% \text { (d.r. 58:42) } \\
& \mathrm{R}=n-\mathrm{Bu}, \mathbf{1 8}, 58 \% \text { (d.r. } 56: 44 \text { ) }
\end{aligned}
$$

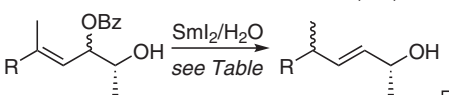

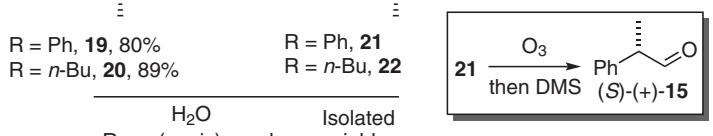

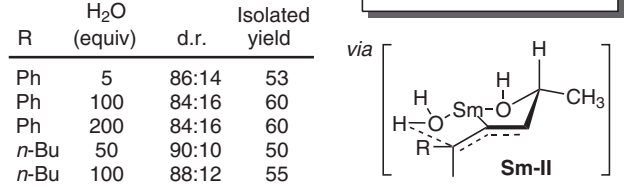

Scheme 5 Synthesis and elimination of lactate-derived compounds $\mathbf{2 1}$ and 22

$\mathrm{yl}$ groups three and four carbons away (as opposed to one and two carbons for compounds $\mathbf{1 9}$ and 12, respectively) from the allylic benzoate position (Scheme 6). The reduction of $\mathbf{2 5}$ proceeded with comparable diastereoselectivity (78:22) to compound 12, giving $\mathbf{2 6}$ via a mechanism presumably involving the 6-membered chelate Sm-III. The methyl group in Sm-III assumes a preferred equatorial conformation, controlling the facial selectivity of the protonation event and explaining the stereochemistry observed in the final product. It was anticipated that the reaction of compound $\mathbf{3 0}$ would give lower selectivity as the 7 -membered

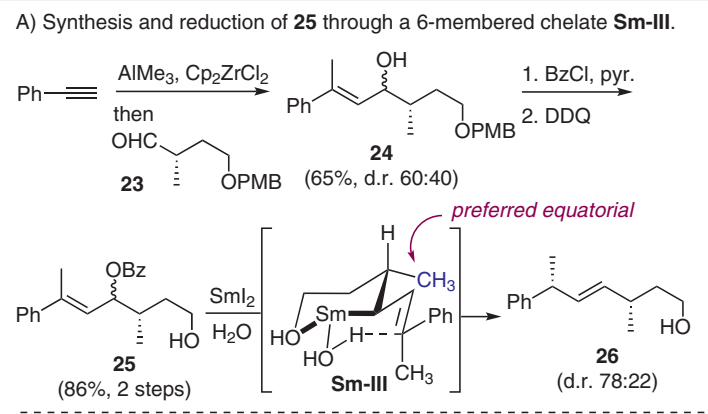

B) Synthesis and reduction of $\mathbf{2 6}$ through a 7-membered chelate Sm-IV.

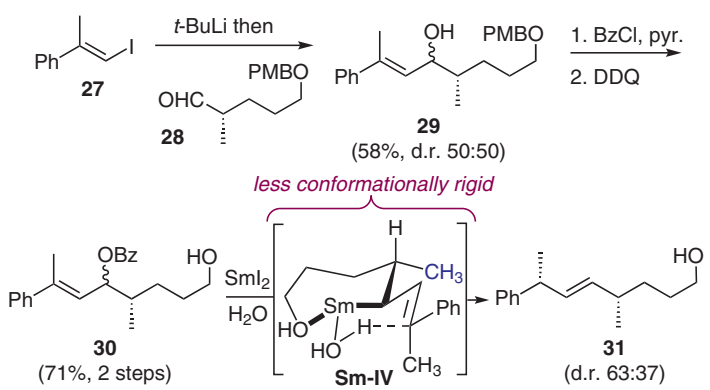

Scheme 6 Comparison of chelation size effects on $\mathrm{Sml}_{2}\left(\mathrm{H}_{2} \mathrm{O}\right)_{n}$ allylic benzoate reductions. The reaction of $\mathbf{3 0}$ (B) gave lower diastereoselectivity than compound $\mathbf{2 5}(\mathrm{A})$, presumably due to formation of a less conformationally rigid 7-membered organosamarium chelate Sm-IV. 
organosamarium ring chelate $\mathbf{S m}$-IV would be less stable and/or less conformationally rigid than 5- and 6-membered chelate substrates. ${ }^{28}$ Indeed, the reaction of $\mathbf{3 0}$ with $\mathrm{SmI}_{2}\left(\mathrm{H}_{2} \mathrm{O}\right)_{\mathrm{n}}$ gave 31 with not only a lower diastereomeric ratio (63:37), but was accompanied by large amounts of what we have assigned as radical dimers. ${ }^{22}$ This result indicates that favorable chelation not only improves d.r., but also controls the product selectivity in these reactions.

Within the 5- and 6-membered chelate series we also set out to evaluate the impact of stereodirecting group location. To that end, several additional substrates 38a-c were prepared (Scheme 7). Combined with compounds 12 and 25, we obtained data for $\mathrm{SmI}_{2}\left(\mathrm{H}_{2} \mathrm{O}\right)_{\mathrm{n}}$ reductions for all permutations of compounds proceeding through 5- or 6-membered chelates containing an $\alpha, \beta$, or $\gamma$ methyl stereodirecting group (Table 2). From these results certain trends emerged. For instance, comparing results for compounds $\mathbf{1 2}$ and 38a (Table 2, entries 2 and 4) indicates that shifting the stereocenter away from the allylic benzoate position results in a slight loss of diastereoselectivity (75:25 for 12 vs 70:30 for 38a) with essentially no change in regioselectivity. This could potentially be explained by the difference between the primary alcohol in $\mathbf{1 2}$ and a secondary alcohol in 38a, with a more sterically hindered alcohol resulting in a loss of samarium chelation and therefore a less conformationally restricted transition state. However, a similar shift of the methyl group in the 6-membered chelate system (entries 3 and 5 ) resulted in a significant loss of diastereoselectivity (78:22 for $\mathbf{2 5}$ vs 57:43 for $\mathbf{3 8 b}$ ). This result cannot be explained by a change in the strength of the chelating group as both $\mathbf{2 5}$ and $\mathbf{3 8 b}$ contain primary alcohols. Rather, it seems that having the stereodirecting group closest to the allylic benzoate position (and thus the resulting carbonbound samarium) is optimal for maximizing the diastereoselectivity of this reaction. Further evidence is provided from the reduction of compound 38c (entry 6), with the stereodirecting methyl group now further remote, and the reaction giving low (and essentially identical to compound 38b) diastereoselectivity.

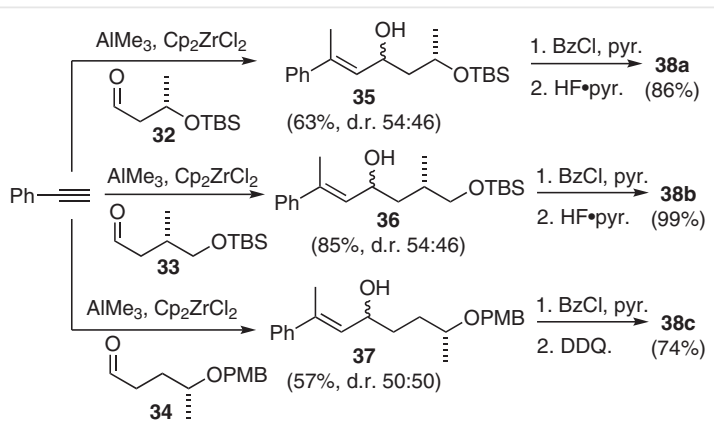

Scheme 7 Synthesis of additional elimination substrates 37 a-c containing differing methyl stereocenter positioning

Table 2 Comparison of Chelation Size and Methyl Stereocenter Position on Regio- and Diastereoselectivity for $\mathrm{Sml}_{2}\left(\mathrm{H}_{2} \mathrm{O}\right)_{n}$ Allylic Benzoate Reductions ${ }^{\mathrm{a}}$

$$
\text { 12, 25,38a-c}
$$

Entry


Ozonolysis of the product mixtures obtained from the reductions of compounds $\mathbf{2 5}$ and 38a produced oppositely enantioenriched mixtures of aldehyde $\mathbf{1 5}$. From 25 we obtained primarily $S-(+)-\mathbf{1 5}$ whereas $\mathbf{3 8 a}$ gave primarily the $R$-(-)-enantiomer. ${ }^{23}$ Both results are consistent with the formation of chelated organosamarium transition state structures Sm-III and Sm-IV, with the methyl group (Me) assuming a preferred equatorial position (Scheme 8).
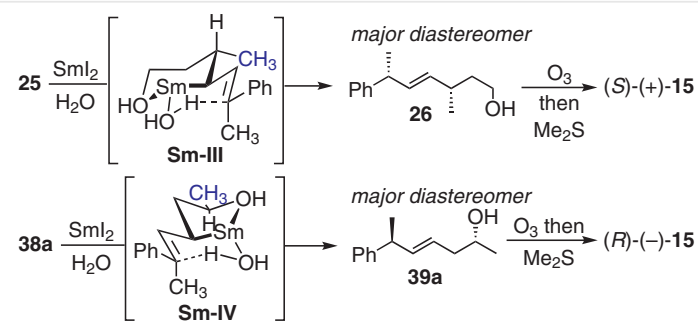

Scheme 8 Determination of absolute stereochemistry for the major diastereomers produced from the reactions of compounds $\mathbf{2 5}$ and $\mathbf{3 8 a}$ and models Sm-III and Sm-IV to explain the outcome

We also prepared a series of compounds $\mathbf{5 0 a}-\mathbf{e}$ in order to examine the effect of stereodirecting group identity in these reactions (Scheme 9, Table 3). It was hypothesized that larger groups might impart better diastereoselectivities based on, for instance, larger energy differences between axial and equatorial conformations. With the exception of substrates containing a hydroxyl (50e, entry 6) or phenyl (50c, entry 4) stereodirecting group where elimination was an issue, all other substrates gave the desired products in good yield and diastereoselectivity. Increasing the size of the stereodirecting group appears to play a modest role in the diastereoselectivity of the reaction. For instance, a change in stereocenter identity from methyl (12, entry 1 ) to isopropyl (50a, entry 2 ) resulted in an increase in diastereoselectivity from 75:25 to 83:17; however, incorporation of an even larger tert-butyl group (50b, entry 3) showed no further increase but rather a small drop in diastereoselectivity (80:20). Reduction of the substrate 50c containing a phenyl stereocenter (entry 4 ) gave the product with a d.r. similar to that of a methyl stereodirecting group (73:27 vs $75: 25$ ) but with a lower isolated yield $(25 \%)$ due to competing elimination to form the fully conjugated diene. The use of a benzyl (Bn) stereocenter (50d, entry 5) resulted in a d.r. similar to that obtained for an $i$-Pr group (81:19). Regioselectivity for all reactions was high (from 93:7 to 100:0) suggestive of a dominant intramolecular protonation pathway.

Based on the results in Tables 2 and 3, we sought to design an optimized substrate for maximizing diastereoselectivity in our $\mathrm{SmI}_{2}\left(\mathrm{H}_{2} \mathrm{O}\right)_{n}$ allylic benzoate reductions. For instance, comparing results for compounds $\mathbf{1 2}$ and $\mathbf{2 5}$ (Table 2 , entries 2 and 3 ) indicates a slightly higher d.r. from a 6membered ring chelated organosamarium intermediate over a 5-membered ring chelate. Additionally, we also observed an enhancement in d.r. by incorporation of $i$-Pr or Bn-stereodirecting groups (Table 3, entries 2 and 5). Combining these effects we thought it might therefore lead to even further enhanced d.r. while still maintaining high yield and regioselectivity. In order to test this hypothesis, we synthesized the 6-membered chelate Bn-stereocenter containing substrate $\mathbf{5 6}$ (Scheme 10). The synthesis began by alkylation of oxazolidinone $\mathbf{5 2}^{29}$ with benzyl bromide giving $\mathbf{5 3}$ in $62 \%$ yield as a single diastereomer after chromatography on silica gel. DIBAL-H reduction of $\mathbf{5 3}$ gave aldehyde $\mathbf{5 4}$ to which was then added the lithium anion generated from vinyl iodide $\mathbf{2 7}^{30}$ by lithium-halogen exchange. This reaction produced secondary alcohol $\mathbf{5 5}$ as a 62:38 mixture of diastereomers in $93 \%$ yield. Benzoylation followed by deprotection of the TBS ether using HF.pyr then gave the final allylic benzoate 'optimized substrate' $\mathbf{5 6}$ in $81 \%$ over the two steps.

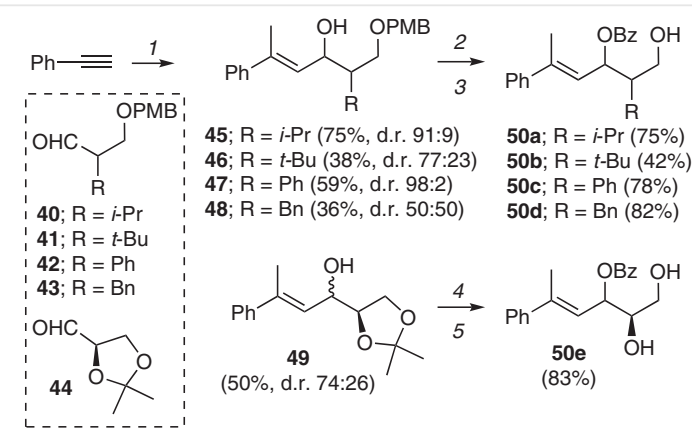

Scheme 9 Reagents and conditions: (1) $\mathrm{AlMe}_{3}, \mathrm{Cp}_{2} \mathrm{ZrCl}$, then 40, 41, 42, 43, or 44; (2) BzCl, pyridine; (3) DDQ; (4) BzCl, pyridine; (5) pTSA, $\mathrm{MeOH}$.

Table 3 Stereodirecting Group Identity Effects on Regio- and Diastereoselectivity for $\mathrm{Sml}_{2}\left(\mathrm{H}_{2} \mathrm{O}\right)_{n}$ Allylic Benzoate Reductions ${ }^{\mathrm{a}}$

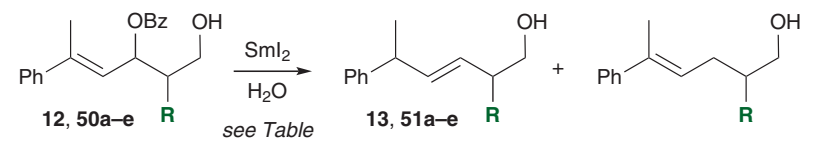

\begin{tabular}{|c|c|c|c|c|}
\hline Entry & $R$ & d.r. ${ }^{b}$ & r.r. ${ }^{b}$ & Yield (\%) \\
\hline 1 & Me (12) & $75: 25$ & $98: 2$ & 90 \\
\hline 2 & $i-\operatorname{Pr}(\mathbf{5 0 a})$ & $83: 17$ & $93: 7$ & 80 \\
\hline 3 & $t$-Bu (50b) & $80: 20$ & $95: 5$ & 73 \\
\hline 4 & $\mathrm{Ph}(\mathbf{5 0 c})$ & $73: 27$ & 100:0 & 25 \\
\hline 5 & $B n(50 d)$ & $81: 19$ & $96: 4$ & 82 \\
\hline 6 & $\mathrm{OH}(\mathbf{5 0 e})$ & - & - & 0 \\
\hline
\end{tabular}

${ }^{a}$ All reductions were performed at r.t. using 7 equiv of $\mathrm{Sml}_{2}$ and 105 equiv of $\mathrm{H}_{2} \mathrm{O}$.

${ }^{\mathrm{b}}$ Determined by ${ }^{1} \mathrm{H}$ NMR analysis.

' Isolated yield. 


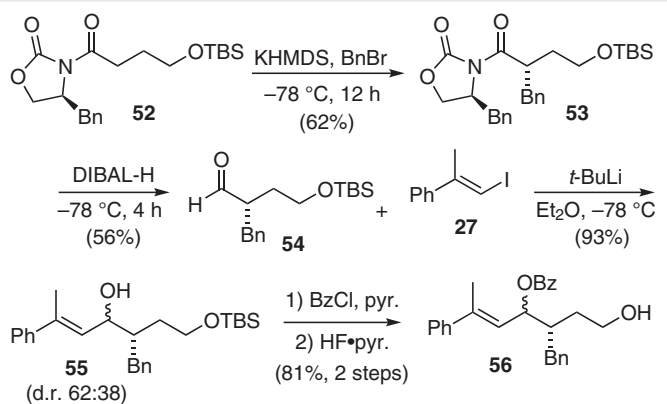

Scheme 10 Synthesis of an optimized substrate $\mathbf{5 6}$ based on results from Tables 2 and 3

Surprisingly upon reduction of $\mathbf{5 6}$ with $\mathrm{SmI}_{2}\left(\mathrm{H}_{2} \mathrm{O}\right)_{n}$, the expected product $\mathbf{5 7}$ was obtained in only $32 \%$ yield with a d.r. of 74:26 and as a 69:31 mixture of regioisomers (r.r., Scheme 11). The low yield was due in part to a significant level of side-product formation (e.g., radical dimers), which were observed in the ${ }^{1} \mathrm{H}$ NMR spectrum of the crude reaction mixture. Other studies have shown that larger ions such as samarium prefer smaller ring systems. ${ }^{31}$ This may explain the higher diastereo- and regioselectivity obtained with the lactate derived substrate $\mathbf{2 0}(90: 10)$ as it had the smallest ring chelate size (nominal 4-membered ${ }^{32}$ ). Additionally as the stereodirecting group becomes larger, steric strain may be introduced into the rigid chelated organosamarium Sm-V. Formation of the 6-membered chelate $\mathbf{S m}-\mathbf{V}$ from reduction of $\mathbf{5 6}$ may therefore not be as favorable, leading to a greater percentage of side-products and lower diastereo- and regioselectivity. Nonetheless, sufficient amounts of $\mathbf{5 7}$ were obtained to determine its absolute configuration. Ozonolysis of $\mathbf{5 7}$ followed by reduction with $\mathrm{NaBH}_{4}$ gave (S)-(-)-5833 indicating the absolute stereochemistry of the major diastereomer of $\mathbf{5 7}$ is $(3 S, 6 R)$. This is consistent with a mechanism involving the 6-6 bicyclic organosamarium transition state structure $\mathbf{S m}-\mathbf{V}$, with the benzyl stereodirecting group occupying a preferred equatorial position, followed by intramolecular proton delivery from a samarium bound water.

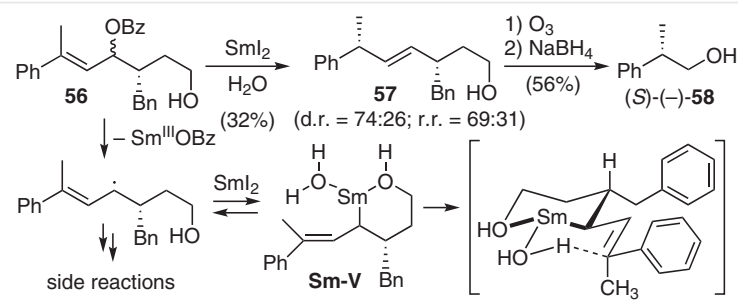

Scheme 11 Results from the $\mathrm{Sml}_{2}\left(\mathrm{H}_{2} \mathrm{O}\right)_{n}$ reduction of an 'optimized' substrate 56 . The d.r. and regioselectivity (r.r.) obtained suggest that the trends observed in Tables 2 and 3 may not be additive.

In summary, samarium-mediated allylic benzoate reductions can occur diastereoselectively when adjacent to a trisubstituted alkene and flanked by a stereodirecting and chelating group. The reaction can achieve high yields, regioselectivity, and diastereoselectivity (up to 90:10). Stereodirecting- and chelating group location appear to have the most significant impact on yield and selectivity in these reactions. Diastereoselectivity tends to increase with shorter chain lengths between the allylic benzoate and the chelating group (e.g., 63:37 d.r. when separated by four carbons vs $90: 10$ when separated by two). However, the highest diastereoselectivity obtained (90:10) by having a chelating hydroxyl immediately adjacent $\alpha$ to the allylic benzoate, was accompanied by competing $\beta$-elimination leading to lower yields. Increasing the size of the stereodirecting group also increased diastereoselectivity, although to a lesser extent (e.g., 75:25 for methyl vs 80:20 for tert-butyl). Combining the results from experiments investigating stereodirecting and chelating group location along with stereodirecting group identity effects, which led to the design and synthesis of an 'optimized substrate' containing an $\alpha$-benzyl stereodirecting group and a hydroxyl group that would generate a 6 -membered ring chelate organosamarium intermediate. Reduction of this compound with $\mathrm{SmI}_{2}\left(\mathrm{H}_{2} \mathrm{O}\right)$, however, proceeded with low yield (32\%) of the desired product and modest diastereoselectivity (74:26). The low yield was the result of moderate regioselectivity (69:31) and the formation of side-products that we assume may include radical dimerization processes. Formation of largerring-chelated organosamarium intermediates containing sterically demanding groups might therefore not be favorable. Models have been proposed to account for these results based on ring-conformation considerations of a chelated organosamarium intermediate and a mechanism involving intramolecular protonation by a samarium-bound water.

All reactions were carried out under $\mathrm{N}_{2}$ in flame-dried glassware, unless otherwise specified. The solvents used were dried by passing the solvent through a column of activated $\mathrm{Al}_{2} \mathrm{O}_{3}$ under $\mathrm{N}_{2}$ immediately prior to use. $\mathrm{SmI}_{2}$ was prepared according to the method of Procter. ${ }^{34}$ All other reagents were purchased and used as received, unless otherwise mentioned. TLC analysis used $0.25 \mathrm{~mm}$ silica gel layer fluorescence $\mathrm{UV}_{254}$ plates. Flash chromatography: silica gel (230-400 mesh). NMR: Spectra were recorded on a Varian Mercury 300 or Bruker 500 spectrometer in the solvents indicated; chemical shifts $(\delta)$ are given in ppm, coupling constants $(J)$ in hertz $(\mathrm{Hz})$. The solvent signals were used as references $\left(\mathrm{CDCl}_{3}: \delta_{\mathrm{c}}=77.0\right.$; residual $\mathrm{CHCl}_{3}$ in $\mathrm{CDCl}_{3}: \delta_{\mathrm{H}}=$ 7.26). MS (EI): Bruker MaXis Impact mass spectrometer. Spectral data listed are for stereoisomeric mixtures unless specifically labelled to the contrary.

\section{Zirconium-Catalyzed Carboalumination; General Procedure}

To a Schlenk tube filled with DCM (0.3 M relative to alkyne) and $\mathrm{Cp}_{2} \mathrm{ZrCl}_{2}$ ( 0.1 equiv) at $-20{ }^{\circ} \mathrm{C}$ was added $\mathrm{AlMe}_{3}$ (2.0 equiv) dropwise resulting in a yellow solution, which was stirred for $10 \mathrm{~min}$. Deionized $\mathrm{H}_{2} \mathrm{O}$ (1.0 equiv) was then added dropwise turning the solution a darker shade of yellow, which was then stirred for another $10 \mathrm{~min}$. The 
reaction was then warmed to r.t. for $10 \mathrm{~min}$ and then cooled to $0{ }^{\circ} \mathrm{C}$. Phenylacetylene (1.0 equiv) was added dropwise and the solution was stirred for $40 \mathrm{~min}$ at $0{ }^{\circ} \mathrm{C}$. The aldehyde ( 0.8 equiv) was then added dropwise and the mixture was stirred for $1 \mathrm{~h}$ at $0{ }^{\circ} \mathrm{C}$. The reaction was quenched slowly with cold $\mathrm{H}_{2} \mathrm{O}$ and then aq $\mathrm{HCl}$, and extracted with $\operatorname{DCM}(3 \times)$. The combined organic extracts were dried $\left(\mathrm{MgSO}_{4}\right)$ and concentrated in vacuo.

\section{Benzoylation of Alcohols; General Procedure}

Pyridine (2 equiv) was added to a Schlenk tube containing substrate ( 1 equiv) in DCM ( $0.2 \mathrm{M}$ relative to substrate). The mixture was then cooled to $0{ }^{\circ} \mathrm{C}$ followed by the addition of benzoyl chloride (1.2 equiv). The reaction was allowed to warm to r.t. for $15 \mathrm{~h}$., before quenching with aq $\mathrm{NaHCO}_{3}$ and extracting with $\mathrm{DCM}(3 \times)$. The combined organic extracts were dried $\left(\mathrm{MgSO}_{4}\right)$ and concentrated in vacuo.

\section{DDQ Removal of a PMB; General Procedure}

Substrate was added to a round-bottomed flask containing a 50:50 mixture of DCM:pH 7 buffer ( $0.1 \mathrm{M}$ relative to substrate). The reaction mixture was cooled to $0{ }^{\circ} \mathrm{C}$ and stirred vigorously at which time DDQ (3 equiv) was added portionwise over $30 \mathrm{~min}$. The reaction was stirred vigorously for $1 \mathrm{~h}$ and then quenched with aq $\mathrm{NaOH}(1.0 \mathrm{M})$ and extracted with DCM $(3 \times)$. The combined organic extracts were washed with brine $(2 \times)$, dried $\left(\mathrm{MgSO}_{4}\right)$, and concentrated in vacuo.

\section{$\mathrm{SmI}_{2}\left(\mathrm{H}_{2} \mathbf{O}\right)_{n}$ Reductions; General Procedure}

To a dry Schlenk tube containing a solution of $\mathrm{SmI}_{2}$ in THF $(0.1 \mathrm{M}, 7$ equiv) was added degassed nano-pure $\mathrm{H}_{2} \mathrm{O}$ (105 equiv) turning the solution to a deep red color. The solution was stirred for $5 \mathrm{~min}$ before the substrate ( 1 equiv) was then added. After $30 \mathrm{~min}$, the reaction was quenched with aq $\mathrm{NaHCO}_{3}$ and extracted with EtOAc $(3 \times)$. The combined organic extracts were dried $\left(\mathrm{MgSO}_{4}\right)$, and concentrated in vacuo.

\section{(E)-2,5-Diphenylhex-4-en-3-yl Benzoate (5)}

Prepared according to the general benzoylation procedure using $(E)$ 2,5-diphenylhex-4-en-3-ol ${ }^{35}$ ( $\left.0.5 \mathrm{~g}, 1.98 \mathrm{mmol}\right)$. Purification by flash chromatography on silica gel gave $\mathbf{5}(0.64 \mathrm{~g}, 90 \%)$ as a colorless oil (d.r. $=80: 20) ; R_{f}=0.52$ (4:1 hexanes:EtOAc).

IR (ATR): 3059, 3028, 2970, 1712, 1601, 1584, 1494, 1450, 1377, 1265 , $998,864,710,696 \mathrm{~cm}^{-1}$.

\section{Major Diastereomer}

${ }^{1} \mathrm{H} \mathrm{NMR}\left(\mathrm{CDCl}_{3}, 500 \mathrm{MHz}\right): \delta=8.08$ (ddd, $\left.J=8.2,3.2,1.9 \mathrm{~Hz}, 2 \mathrm{H}\right), 7.57$ (dd, $J=6.8,1.3 \mathrm{~Hz}, 1 \mathrm{H}), 7.46(\mathrm{t}, J=7.8 \mathrm{~Hz}, 2 \mathrm{H}), 7.44-7.33(\mathrm{~m}, 2 \mathrm{H})$, 7.33-7.26 (m, 5 H), 7.26-7.20 (m, $3 \mathrm{H}), 6.01(\mathrm{dd}, J=9.4,7.5 \mathrm{~Hz}, 1 \mathrm{H})$, $5.64(\mathrm{dq}, J=9.3,1.4 \mathrm{~Hz}, 1 \mathrm{H}), 3.29(\mathrm{dq}, J=7.1 \mathrm{~Hz}, 1 \mathrm{H}), 2.01(\mathrm{~d}, J=1.4$ $\mathrm{Hz}, 3 \mathrm{H}), 1.47$ (d, J = 7.0 Hz, $3 \mathrm{H}$ ).

${ }^{13} \mathrm{C} \mathrm{NMR}\left(\mathrm{CDCl}_{3}, 126 \mathrm{MHz}\right): \delta=165.97,143.02,142.29,139.89$, $132.94,130.60,129.68,128.42,128.41,128.27,128.19,127.35$, 126.79, 125.99, 124.75, 76.04, 44.62, 17.16, 16.72.

HRMS (ES+): $m / z$ [379.1674] $]^{+}$calcd for $\mathrm{C}_{25} \mathrm{H}_{24} \mathrm{O}_{2} \mathrm{Na}^{+}[\mathrm{M}+\mathrm{Na}]^{+}$; found: 379.1640 .

\section{(2S,E)-1-[(4-Methoxybenzyl)oxy]-2-methyl-5-phenylhex-4-en-3-} ol (8)

Prepared according to the general $\mathrm{Zr}$-catalyzed carboalumination procedure using aldehyde $7^{17}(1.0 \mathrm{~g}, 4.7 \mathrm{mmol})$. Purification by flash chromatography on silica gel gave $\mathbf{8}(1.31 \mathrm{~g}, 85 \%)$ as a colorless oil (d.r. $=70: 30) ; R_{f}=0.65$ ( $1: 1$ hexanes:EtOAc).

IR (ATR): 3320, 3028, 2986, 2962, 2851, 1713, 1611, 1595, 1576, 1440 , $1246,1035,699 \mathrm{~cm}^{-1}$.

${ }^{1} \mathrm{H} \mathrm{NMR}\left(\mathrm{CDCl}_{3}, 500 \mathrm{MHz}\right): \delta=7.43(\mathrm{~d}, J=7.1 \mathrm{~Hz}, 2 \mathrm{H}), 7.34(\mathrm{t}, J=7.3$ $\mathrm{Hz}, 2 \mathrm{H}), 7.29(\mathrm{t}, J=8.7 \mathrm{~Hz} 3 \mathrm{H}), 6.91(\mathrm{~d}, J=8.6 \mathrm{~Hz}, 2 \mathrm{H}), 5.78(\mathrm{dq}, J=$ $8.9,1.4 \mathrm{~Hz}, 1 \mathrm{H}), 4.50(\mathrm{~d}, J=11.7 \mathrm{~Hz}, 1 \mathrm{H}), 4.51(\mathrm{~m}, 1 \mathrm{H}), 4.47(\mathrm{~d}, J=$ $11.7 \mathrm{~Hz}, 1 \mathrm{H}), 3.83(\mathrm{~s}, 3 \mathrm{H}), 3.66(\mathrm{dd}, J=9.3,4.3 \mathrm{~Hz}, 1 \mathrm{H}), 3.51(\mathrm{dd}, J=$ 9.3, 7.6 Hz, $1 \mathrm{H}), 2.12(\mathrm{~d}, J=1.4 \mathrm{~Hz}, 3 \mathrm{H}), 2.03(\mathrm{qd}, J=7.4,4.3 \mathrm{~Hz}, 1 \mathrm{H})$. $0.93(\mathrm{~d}, J=7.1 \mathrm{~Hz}, 3 \mathrm{H})$.

${ }^{13} \mathrm{C}$ NMR $\left(\mathrm{CDCl}_{3}, 126 \mathrm{MHz}\right): \delta=159.27,143.22,137.42,129.87$, 129.50, 129.34, 128.15, 127.10, 125.88, 113.84, 74.49, 73.11, 73.10, $55.25,39.34,16.52,13.45$.

HRMS (ES+): $m / z$ [349.1780] $]^{+}$calcd for $\mathrm{C}_{21} \mathrm{H}_{26} \mathrm{O}_{3} \mathrm{Na}^{+}[\mathrm{M}+\mathrm{Na}]^{+}$; found: 349.1771 .

\section{(2S,E)-1-[(4-Methoxybenzyl)oxy]-2-methyl-5-phenylhex-4-en-3-yl Benzoate (9)}

Prepared according to the general benzoylation procedure using 8 $(1.31 \mathrm{~g}, 4.00 \mathrm{mmol})$. Purification by flash chromatography on silica gel gave 9 (1.72 g, quant.) as a colorless oil; $R_{f}=0.48$ (4:1 hexanes: EtOAc).

IR (ATR): 3063, 3032, 2999, 2962, 2934, 2917, 2851, 1786, 1713, 1611 , 1599, 1584, 1450, 1246, 1035, $699 \mathrm{~cm}^{-1}$.

${ }^{1} \mathrm{H} \mathrm{NMR}\left(\mathrm{CDCl}_{3}, 500 \mathrm{MHz}\right): \delta=8.02(\mathrm{dd}, J=7.0,1.3 \mathrm{~Hz}, 2 \mathrm{H}), 7.54(\mathrm{dd}$, $J=8.2,7.6 \mathrm{~Hz}, 2 \mathrm{H}), 7.42(\mathrm{t}, J=8.0 \mathrm{~Hz}, 2 \mathrm{H}), 7.38(\mathrm{t}, J=7.0 \mathrm{~Hz}, 2 \mathrm{H}), 7.31$ $(\mathrm{t}, J=7.2 \mathrm{~Hz}, 2 \mathrm{H}), 7.25-7.21(\mathrm{~m}, 2 \mathrm{H}), 6.81(\mathrm{~d}, J=8.0 \mathrm{~Hz}, 2 \mathrm{H}), 5.96$ (dd, $J=9.5,6.8 \mathrm{~Hz}, 1 \mathrm{H}), 5.77(\mathrm{dq}, J=9.5,1.4 \mathrm{~Hz}, 1 \mathrm{H}), 4.45(\mathrm{~d}, J=11.7 \mathrm{~Hz}, 1$ $\mathrm{H}), 4.40(\mathrm{~d}, J=11.7 \mathrm{~Hz}, 1 \mathrm{H}), 3.77(\mathrm{~s}, 3 \mathrm{H}), 3.49(\mathrm{t}, J=7.0 \mathrm{~Hz}, 1 \mathrm{H}), 3.44$ (dd, $J=6.0,9.2 \mathrm{~Hz}, 1 \mathrm{H}), 2.35$ (hept, $J=6.9 \mathrm{~Hz}, 1 \mathrm{H}), 2.27$ (d, $J=1.3 \mathrm{~Hz}$, $3 \mathrm{H}), 1.11(\mathrm{~d}, J=7.0 \mathrm{~Hz}, 3 \mathrm{H})$.

${ }^{13} \mathrm{C}$ NMR $\left(\mathrm{CDCl}_{3}, 126 \mathrm{MHz}\right): \delta=165.73,159.06,143.00,140.35$, $134.54,132.73,130.59,129.59,129.24,128.89,128.29,128.21$, $127.40,126.00,124.06,113.70,73.37,72.79,71.60,55.23,38.33$, $16.81,13.09$.

HRMS (ES+): $m / z[453.2042]^{+}$calcd for $\mathrm{C}_{28} \mathrm{H}_{30} \mathrm{O}_{4} \mathrm{Na}^{+}[\mathrm{M}+\mathrm{Na}]^{+}$; found: 453.2039.

\section{1-Methoxy-4-(\{[(2R,E)-2-methyl-5-phenylhex-3-en-1-} yl]oxy\}methyl)benzene (10)

To a dry Schlenk flask containing a solution of $\mathrm{SmI}_{2}$ in $\operatorname{THF}(0.1 \mathrm{M}$, $16.1 \mathrm{~mL})$ at $0{ }^{\circ} \mathrm{C}$ was added DMPU $(0.445 \mathrm{~mL}, 1.61 \mathrm{mmol})$ resulting in a dark purple solution, which was stirred for $1 \mathrm{~h}$. Compound $\mathbf{9}(0.100$ $\mathrm{g}, 0.23 \mathrm{mmol}$ ) was then added and the solution was stirred for $1 \mathrm{~h}$. The reaction was then quenched with aq $\mathrm{NH}_{4} \mathrm{Cl}(20 \mathrm{~mL})$ and extracted with EtOAc $(3 \times 20 \mathrm{~mL})$. The combined organic extracts were dried $\left(\mathrm{MgSO}_{4}\right)$, and concentrated in vacuo. Purification by flash chromatography on silica gel gave $\mathbf{1 0}(0.051 \mathrm{~g}, 70 \%)$ as a colorless oil (d.r. = $60: 40) ; R_{f}=0.60$ (10:1 hexanes:EtOAc).

IR (ATR): 3080, 3057, 3025, 2957, 2926, 2850, 1948, 1877, 1804, 1730 , $1611,1511,1452,1360,1245,1087,1035,819,757,698 \mathrm{~cm}^{-1}$.

${ }^{1} \mathrm{H}$ NMR $\left(\mathrm{CDCl}_{3}, 500 \mathrm{MHz}\right): \delta=7.29(\mathrm{t}, J=7.5 \mathrm{~Hz}, 4 \mathrm{H}), 7.20(\mathrm{dd}, J=$ $11.1,2.3 \mathrm{~Hz}, 4 \mathrm{H}), 7.17(\mathrm{t}, J=6.7 \mathrm{~Hz}, 2 \mathrm{H}), 6.87(\mathrm{~d}, J=8.5 \mathrm{~Hz}, 4 \mathrm{H}), 6.86$ (d, $J=8.6 \mathrm{~Hz}, 4 \mathrm{H}$ ), 5.63 (ddd, $J=15.5,6.7,1.3 \mathrm{~Hz}, 2 \mathrm{H}$ ), 5.39 (ddd, $J=$ 15.5, 7.1, $1.4 \mathrm{~Hz}, 2 \mathrm{H}$ ), 4.42 (d, $J=6.53 \mathrm{~Hz}, 4 \mathrm{H}), 3.79$ (s, $3 \mathrm{H}), 3.78(\mathrm{~s}, 3$ $\mathrm{H}), 3.43$ (pent, $J=7.0 \mathrm{~Hz}, 2 \mathrm{H}$ ), $3.32(\mathrm{dd}, J=9.2,6.2 \mathrm{~Hz}, 1 \mathrm{H}), 3.31$ (dd, $J=9.1,6.3 \mathrm{~Hz}, 1 \mathrm{H}), 3.24(\mathrm{dd}, J=9.2,6.2 \mathrm{~Hz}, 1 \mathrm{H}), 3.23(\mathrm{dd}, J=7.1,4.0$ $\mathrm{Hz}, 1 \mathrm{H}), 2.48$ (hept, $J=6.7 \mathrm{~Hz}, 2 \mathrm{H}), 1.31(\mathrm{~d}, J=7.0 \mathrm{~Hz}, 3 \mathrm{H}), 1.00(\mathrm{~d}, J=$ $6.8 \mathrm{~Hz}, 3 \mathrm{H}) .0 .99$ (d, $J=6.6 \mathrm{~Hz}, 3 \mathrm{H})$. 
${ }^{13} \mathrm{C}$ NMR $\left(\mathrm{CDCl}_{3}, 126 \mathrm{MHz}\right): \delta=159.04,134.62,131.63,129.13$, 129.08, 128.30, 128.10, 127.18, 125.89, 113.70, 75.14, 72.50, 55.23, $42.20,36.76,21.48,17.12$.

HRMS (ES+): $m / z$ [333.1830] $]^{+}$calcd for $\mathrm{C}_{21} \mathrm{H}_{26} \mathrm{O}_{2} \mathrm{Na}^{+}[\mathrm{M}+\mathrm{Na}]^{+}$; found: 333.1836.

\section{(2S,E)-1-Hydroxy-2-methyl-5-phenylhex-4-en-3-yl Benzoate (12)}

Prepared according to the general procedure for removal of a PMB group with DDQ using $9(1.2 \mathrm{~g}, 2.78 \mathrm{mmol})$. Purification by flash chromatography on silica gel gave $\mathbf{1 2}(0.6 \mathrm{~g}, 70 \%)$ as a colorless oil; $R_{f}=$ 0.18 (4:1 hexanes:EtOAc).

IR (ATR): 3420, 3060, 3032, 2964, 2922, 2880, 1714, 1450, 1268, 1110 , $932,711 \mathrm{~cm}^{-1}$.

\section{Major Diastereomer}

${ }^{1} \mathrm{H} \mathrm{NMR}\left(\mathrm{CDCl}_{3}, 500 \mathrm{MHz}\right): \delta=8.07(\mathrm{dd}, J=8.3,1.2 \mathrm{~Hz}, 2 \mathrm{H}), 7.57(\mathrm{t}, J=$ $7.4 \mathrm{~Hz}, 1 \mathrm{H}), 7.45(\mathrm{t}, J=7.7 \mathrm{~Hz}, 2 \mathrm{H}), 7.42(\mathrm{dd}, J=7.2,1.3 \mathrm{~Hz}, 2 \mathrm{H}), 7.33$ $(\mathrm{t}, J=7.7 \mathrm{~Hz}, 2 \mathrm{H}), 7.28(\mathrm{t}, J=7.2 \mathrm{~Hz}, 1 \mathrm{H}), 5.95(\mathrm{dd}, J=9.4,8.1 \mathrm{~Hz}, 1 \mathrm{H})$, $5.84(\mathrm{dq}, J=9.4,1.4 \mathrm{~Hz}, 1 \mathrm{H}), 3.68(\mathrm{qd}, J=11.3,4.6 \mathrm{~Hz}, 2 \mathrm{H}), 2.23(\mathrm{~d}, J=$ $1.4 \mathrm{~Hz}, 3 \mathrm{H}), 2.15(\mathrm{~m}, 1 \mathrm{H}), 1.10(\mathrm{~d}, J=7.0 \mathrm{~Hz}, 3 \mathrm{H})$.

${ }^{13} \mathrm{C}$ NMR $\left(\mathrm{CDCl}_{3}, 126 \mathrm{MHz}\right): \delta=166.48,142.73,140.69,133.05$, 130.20, 129.67, 128.38, 128.25, 127.54, 125.96, 124.33, 73.31, 64.09, 40.55, 16.88, 12.92 .

HRMS (ES+): $m / z[333.1467]^{+}$calcd for $\mathrm{C}_{21} \mathrm{H}_{26} \mathrm{O}_{2} \mathrm{Na}^{+}[\mathrm{M}+\mathrm{Na}]^{+}$; found: 333.1472 .

\section{(2R,5R,E)-2-Methyl-5-phenylhex-3-en-1-ol (13)}

Prepared according to the general procedure for $\mathrm{SmI}_{2}\left(\mathrm{H}_{2} \mathrm{O}\right)_{n}$ reductions using compound 12 (0.025 g, $0.08 \mathrm{mmol})$. Purification by flash chromatography on silica gel gave $\mathbf{1 5}(0.0135 \mathrm{~g}, 90 \%)$ as a pale yellow oil; $R_{f}=0.31$ ( $4: 1$ hexanes:EtOAc).

IR (ATR): 3360, 3083, 3061, 3025, 2961, 2925, 2871, 1950, 1876, 1803 , $1716,1601,1492,1415,1373,1272,1029,971,760,698 \mathrm{~cm}^{-1}$.

\section{Major Diastereomer}

${ }^{1} \mathrm{H} \mathrm{NMR}\left(\mathrm{CDCl}_{3}, 500 \mathrm{MHz}\right): \delta=7.38(\mathrm{t}, J=4.7 \mathrm{~Hz}, 1 \mathrm{H}), 7.30(\mathrm{t}, J=6.9$ $\mathrm{Hz}, 2 \mathrm{H}$ ), 7.20 (d, $J=8.0 \mathrm{~Hz}, 2 \mathrm{H}$ ), 5.74 (ddd, $J=15.5,6.8,1.1 \mathrm{~Hz}, 1 \mathrm{H}$ ), 5.33 (ddd, $J=15.5,7.9,1.4 \mathrm{~Hz}, 1 \mathrm{H}$ ), 3.47 (m, $2 \mathrm{H}$ ), 3.38 (dd, $J=10.6,8.1$ $\mathrm{Hz}, 1 \mathrm{H}), 2.36$ (hept, $J=7.0 \mathrm{~Hz}, 1 \mathrm{H}), 1.36(\mathrm{~d}, J=7.0 \mathrm{~Hz}, 3 \mathrm{H}), 1.01(\mathrm{~d}, J=$ $6.9 \mathrm{~Hz}, 3 \mathrm{H})$.

${ }^{13} \mathrm{C}$ NMR $\left(\mathrm{CDCl}_{3}, 126 \mathrm{MHz}\right): \delta=146.04,136.89,131.00,128.43$, 127.07, 126.06, 67.35, 42.27, 39.66, 21.48, 16.60.

HRMS (ES+): $m / z$ [190.1358 $]^{+}$calcd for $\mathrm{C}_{13} \mathrm{H}_{18} \mathrm{O}^{+}[\mathrm{M}]^{+}$; found: 190.1358 .

\section{(S)-2-Phenylpropanal (15)}

To a round-bottomed flask open to air containing $13,21,26$, or 39 a in $\operatorname{DCM}\left(0.1 \mathrm{M}\right.$ relative to substrate) at $-78{ }^{\circ} \mathrm{C}, \mathrm{O}_{3}$ was bubbled into the solution until the reaction mixture turned to an electric blue color. The reaction was left at this temperature without stirring for $5 \mathrm{~min}$ and then $\mathrm{N}_{2}$ was bubbled though the reaction until the solution became colorless. The reaction was quenched with $\mathrm{Me}_{2} \mathrm{~S}$ (5 equiv), warmed to r.t., and stirred for $1 \mathrm{~h}$. The reaction mixture was washed with brine $(15 \mathrm{~mL})$ and extracted with DCM $(3 \times 15 \mathrm{~mL})$. The combined organic extracts were dried $\left(\mathrm{MgSO}_{4}\right)$ and concentrated in vacuo. Aldehyde $\mathbf{1 5}$ was then isolated by flash chromatography on silica gel; $R_{f}=0.63$ (4:1 hexanes:EtOAc). NMR spectra for $\mathbf{1 5}$ matched with that previously reported. ${ }^{23}$
Polarimetry value for $(S)-\mathbf{1 5}$ from 21: $[\alpha]_{\mathrm{D}}+88.5\left(\right.$ c $\left.0.4, \mathrm{CHCl}_{3}\right)\left\{\mathrm{Lit}^{23}\right.$ for $(R)-15:[\alpha]_{D}-88.6\left(c 0.93, \mathrm{CHCl}_{3}\right)$.

(2R,E)-2-[(4-Methoxybenzyl)oxy]-5-phenylhex-4-en-3-ol (17)

Prepared according to the general procedure for $\mathrm{Zr}$-catalyzed carboaluminations using aldehyde $\mathbf{1 6}^{26}(0.500 \mathrm{~g}, 2.6 \mathrm{mmol})$. Purification by flash chromatography over silica gel gave $17(0.688 \mathrm{~g}, 82 \%)$ as a colorless oil (d.r. $=58: 42) ; R_{f}\left(\right.$ diastereomer $\left._{\alpha}\right)=0.30 ; R_{f}($ diastereo$\left.\operatorname{mer}_{\beta}\right)=0.20$ ( $4: 1$ hexanes:EtOAc).

IR (ATR): 3328, 3058, 3016, 2928, 1268, 1110, 932, $711 \mathrm{~cm}^{-1}$.

\section{Diastereomer $\alpha$}

${ }^{1} \mathrm{H} \mathrm{NMR}\left(\mathrm{CDCl}_{3}, 500 \mathrm{MHz}\right): \delta=7.40(\mathrm{dd}, J=8.6,1.5 \mathrm{~Hz}, 2 \mathrm{H}), 7.33(\mathrm{t}, J=$ $7.1 \mathrm{~Hz}, 2 \mathrm{H}$ ), 7.30 (d, $J=8.7 \mathrm{~Hz}, 2 \mathrm{H}), 7.27$ (t, $J=7.3 \mathrm{~Hz}, 1 \mathrm{H}), 6.90$ (d, $J=$ $8.7 \mathrm{~Hz}, 2 \mathrm{H}), 5.70(\mathrm{dq}, J=8.9,1.4 \mathrm{~Hz}, 1 \mathrm{H}), 4.66(\mathrm{~d}, J=11.3 \mathrm{~Hz}, 1 \mathrm{H})$, $4.43(\mathrm{~d}, J=11.3 \mathrm{~Hz} 1 \mathrm{H}), 4.38(\mathrm{dd}, J=8.9,7.7 \mathrm{~Hz}, 1 \mathrm{H}), 3.82(\mathrm{~s}, 3 \mathrm{H})$, $3.50(\mathrm{dq}, J=7.7,6.2 \mathrm{~Hz}, 1 \mathrm{H}), 2.13(\mathrm{~d}, J=1.4 \mathrm{~Hz}, 3 \mathrm{H}), 1.20(\mathrm{~d}, J=6.2 \mathrm{~Hz}$, $3 \mathrm{H})$.

${ }^{13} \mathrm{C}$ NMR $\left(\mathrm{CDCl}_{3}, 126 \mathrm{MHz}\right): \delta=159.32,142.98,139.81,130.21$, 129.48, 128.18, 127.30, 126.40, 125.88, 113.92, 78.64, 72.44, 70.91, $55.26,16.90,15.52$.

HRMS (ES+): $m / z$ [335.1623 $]^{+}$calcd for $\mathrm{C}_{20} \mathrm{H}_{24} \mathrm{O}_{3} \mathrm{Na}^{+}[\mathrm{M}+\mathrm{Na}]^{+}$; found: 335.1612 .

\section{Diastereomer $\boldsymbol{\beta}$}

${ }^{1} \mathrm{H} \mathrm{NMR}\left(\mathrm{CDCl}_{3}, 500 \mathrm{MHz}\right): \delta=7.40(\mathrm{dd}, J=8.7,1.4 \mathrm{~Hz}, 2 \mathrm{H}), 7.32(\mathrm{t}, J=$ $7.19 \mathrm{~Hz}, 2 \mathrm{H}), 7.29$ (d, $J=8.6 \mathrm{~Hz}, 2 \mathrm{H}), 7.27(\mathrm{t}, J=7.5 \mathrm{~Hz}, 1 \mathrm{H}), 6.89$ (d, $J=8.7 \mathrm{~Hz}, 2 \mathrm{H}), 5.80(\mathrm{dq}, J=8.4,1.3 \mathrm{~Hz}, 1 \mathrm{H}), 4.62(\mathrm{dd}, J=8.4,3.6 \mathrm{~Hz}, 1$ $\mathrm{H}), 4.62(\mathrm{~d}, J=11.7 \mathrm{~Hz}, 1 \mathrm{H}), 4.50(\mathrm{~d}, J=11.7 \mathrm{~Hz}, 1 \mathrm{H}), 3.81(\mathrm{~s}, 3 \mathrm{H})$, $3.66(\mathrm{qd}, J=6.4,3.5 \mathrm{~Hz}, 1 \mathrm{H}), 2.08(\mathrm{~d}, J=1.4 \mathrm{~Hz}, 3 \mathrm{H}), 1.20(\mathrm{~d}, J=6.3 \mathrm{~Hz}$, $3 \mathrm{H})$.

${ }^{13} \mathrm{C}$ NMR $\left(\mathrm{CDCl}_{3}, 126 \mathrm{MHz}\right): \delta=159.26,143.03,138.24,130.57$, $129.53,129.31,128.23,127.26,126.55,125.89,113.88,77.19,70.92$, 70.62, 55.31, 16.56, 14.42 .

HRMS (ES+): $m / z$ [335.1623] $]^{+}$calcd for $\mathrm{C}_{20} \mathrm{H}_{24} \mathrm{O}_{3} \mathrm{Na}^{+}[\mathrm{M}+\mathrm{Na}]^{+}$; found: 335.1612 .

\section{(2R,E)-2-Hydroxy-5-phenylhex-4-en-3-yl Benzoate (19)}

Prepared according to the general benzoylation procedure using $\mathbf{1 7}$ $(0.371 \mathrm{~g}, 1.18 \mathrm{mmol})$. The general procedure for DDQ removal of the PMB was then performed on the crude benzoylation product mixture obtained. Purification by flash chromatography on silica gel gave $\mathbf{1 9}$ $\left(0.281 \mathrm{~g}, 80 \%\right.$ over two steps) as a colorless oil; $R_{f}=0.24$ (4:1 hexanes: EtOAc).

IR (ATR): 3450, 3062, 3031, 2976, 2929, 1712, 1600, 1583, 1450, 1266 , $1110,1025,963,909,709 \mathrm{~cm}^{-1}$.

\section{Diastereomer $\alpha$}

${ }^{1} \mathrm{H} \mathrm{NMR}\left(\mathrm{CDCl}_{3}, 500 \mathrm{MHz}\right): \delta=8.07(\mathrm{dd}, J=8.3,1.2 \mathrm{~Hz}, 2 \mathrm{H}), 7.57(\mathrm{t}, J=$ $7.3 \mathrm{~Hz}, 1 \mathrm{H}), 7.45(\mathrm{t}, J=8.1 \mathrm{~Hz}, 2 \mathrm{H}), 7.41(\mathrm{dd}, J=8.4,1.5 \mathrm{~Hz}, 2 \mathrm{H}), 7.32$ $(\mathrm{t}, J=7.1 \mathrm{~Hz}, 2 \mathrm{H}), 7.27(\mathrm{t}, J=7.3 \mathrm{~Hz}, 1 \mathrm{H}), 5.77(\mathrm{~m}, 2 \mathrm{H}), 4.10$ (pent, $J=$ $6.3 \mathrm{~Hz}, 1 \mathrm{H}), 2.29$ (d, $J=1.2 \mathrm{~Hz}, 3 \mathrm{H}), 1.30(\mathrm{~d}, J=6.4 \mathrm{~Hz}, 3 \mathrm{H})$.

${ }^{13} \mathrm{C} \mathrm{NMR}\left(\mathrm{CDCl}_{3}, 126 \mathrm{MHz}\right): \delta=166.01,142.54,141.90,133.09$, 130.16, 129.66, 128.41, 128.27, 127.68, 125.96, 122.37, 69.74, 18.81, 17.07 .

\section{Diastereomer $\beta$}

${ }^{1} \mathrm{H} \mathrm{NMR}\left(\mathrm{CDCl}_{3}, 500 \mathrm{MHz}\right): \delta=8.07(\mathrm{dd}, J=8.3,1.2 \mathrm{~Hz}, 2 \mathrm{H}), 7.57(\mathrm{t}, J=$ $7.3 \mathrm{~Hz}, 1 \mathrm{H}$ ), 7.45 (t, $J=8.1 \mathrm{~Hz}, 2 \mathrm{H}), 7.43$ (dd, $J=8.4,1.3 \mathrm{~Hz}, 1 \mathrm{H}), 7.33$ 
$(\mathrm{t}, J=7.1 \mathrm{~Hz}, 2 \mathrm{H}), 7.28(\mathrm{t}, J=7.3 \mathrm{~Hz}, 1 \mathrm{H}), 5.91(\mathrm{dq}, J=9.3,1.3 \mathrm{~Hz}, 1 \mathrm{H})$, $5.85(\mathrm{dd}, J=9.3,4.0 \mathrm{~Hz}, 1 \mathrm{H}), 4.15(\mathrm{qd}, J=6.5,4.1 \mathrm{~Hz}, 1 \mathrm{H}), 2.25(\mathrm{~d}, J=$ $1.3 \mathrm{~Hz}, 3 \mathrm{H}), 1.31(\mathrm{~d}, J=6.4 \mathrm{~Hz}, 3 \mathrm{H})$.

${ }^{13} \mathrm{C}$ NMR $\left(\mathrm{CDCl}_{3}, 126 \mathrm{MHz}\right): \delta=165.94,142.47,142.03,133.06$, $130.15,129.64,128.39,128.25,127.66,125.95,122.37,121.60,76.73$, 75.88, 69.59, 18.19, 16.90 .

HRMS (ES+): $m / z$ [319.1310] $]^{+}$calcd for $\mathrm{C}_{19} \mathrm{H}_{20} \mathrm{O}_{3} \mathrm{Na}^{+}[\mathrm{M}+\mathrm{Na}]^{+}$; found: 319.1314

\section{(2R,E)-2-[(4-Methoxybenzyl)oxy]-5-methylnon-4-en-3-ol (18)}

Prepared according to the general procedure for $\mathrm{Zr}$-catalyzed carboaluminations using 1-hexyne $(0.373 \mathrm{~mL}, 3.25 \mathrm{mmol})$ and aldehyde $\mathbf{1 6}^{26}(0.500 \mathrm{~g}, 2.6 \mathrm{mmol})$. Purification by flash chromatography on silica gel gave $18(0.441 \mathrm{~g}, 58 \%)$ as a colorless oil (d.r. $=56: 44) ; R_{f}$ (diastereomer $\left._{\alpha}\right)=0.38 ; R_{f}\left(\right.$ diastereomer $\left._{\beta}\right)=0.34$ (4:1 hexanes:EtOAc).

IR (ATR): 3420, 2980, 2928, 1614, 1570, 1265, 1110, 932, 886, $711 \mathrm{~cm}^{-1}$.

\section{Diastereomer $\boldsymbol{\alpha}$}

${ }^{1} \mathrm{H} \mathrm{NMR}\left(\mathrm{CDCl}_{3}, 500 \mathrm{MHz}\right): \delta=7.29(\mathrm{~d}, J=8.3 \mathrm{~Hz}, 2 \mathrm{H}), 6.91$ (d, $J=8.7$ $\mathrm{Hz}, 2 \mathrm{H}), 5.13(\mathrm{dq}, J=9.0,1.3 \mathrm{~Hz}, 1 \mathrm{H}), 4.64(\mathrm{~d}, J=11.4 \mathrm{~Hz}, 1 \mathrm{H}), 4.42(\mathrm{~d}$, $J=11.3 \mathrm{~Hz}, 1 \mathrm{H}), 4.21(\mathrm{dd}, J=9.0,8.0 \mathrm{~Hz}, 1 \mathrm{H}), 3.82(\mathrm{~s}, 3 \mathrm{H}), 3.39(\mathrm{dq}$, $J=8.0,6.2 \mathrm{~Hz}, 1 \mathrm{H}), 2.04(\mathrm{t}, J=7.7 \mathrm{~Hz}, 2 \mathrm{H}), 1.71(\mathrm{~d}, J=1.4 \mathrm{~Hz}, 3 \mathrm{H})$, $1.42(\mathrm{~m}, 2 \mathrm{H}), 1.31$ (hept, $J=7.3 \mathrm{~Hz}, 2 \mathrm{H}), 1.13(\mathrm{~d}, J=6.2 \mathrm{~Hz}, 3 \mathrm{H}), 0.92$ $(\mathrm{t}, J=7.3 \mathrm{~Hz}, 3 \mathrm{H})$.

${ }^{13} \mathrm{C}$ NMR $\left(\mathrm{CDCl}_{3}, 126 \mathrm{MHz}\right): \delta=159.22,141.64,130.31,129.37$, 123.08, 113.84, 78.91, 72.09, 70.80, 55.20, 39.38, 29.79, 22.29, 16.97, $15.36,13.92$

\section{Diastereomer $\beta$}

${ }^{1} \mathrm{H} \mathrm{NMR}\left(\mathrm{CDCl}_{3}, 500 \mathrm{MHz}\right): \delta=7.27(\mathrm{~d}, J=8.7 \mathrm{~Hz}, 2 \mathrm{H}), 6.88(\mathrm{~d}, J=8.7$ $\mathrm{Hz}, 2 \mathrm{H}), 5.20(\mathrm{dq}, J=8.5,1.3 \mathrm{~Hz}, 1 \mathrm{H}), 4.57(\mathrm{~d}, J=11.5 \mathrm{~Hz}, 1 \mathrm{H}), 4.47$ (d, $J=11.1 \mathrm{~Hz}, 1 \mathrm{H}), 4.46(\mathrm{dd}, J=8.3,3.9 \mathrm{~Hz}, 1 \mathrm{H}), 3.81(\mathrm{~s}, 3 \mathrm{H}), 3.55(\mathrm{qd}$, $J=6.4,3.4 \mathrm{~Hz}, 1 \mathrm{H}), 2.01(\mathrm{t}, J=7.0 \mathrm{~Hz}, 2 \mathrm{H}), 1.64(\mathrm{~d}, J=1.4 \mathrm{~Hz}, 3 \mathrm{H})$, 1.39 (pent, $J=7.5 \mathrm{~Hz}, 2 \mathrm{H}), 1.29(\mathrm{~m}, 2 \mathrm{H}), 1.12(\mathrm{~d}, J=6.4 \mathrm{~Hz}, 3 \mathrm{H}), 0.89$ $(\mathrm{t}, J=7.3 \mathrm{~Hz}, 3 \mathrm{H})$.

${ }^{13} \mathrm{C}$ NMR $\left(\mathrm{CDCl}_{3}, 126 \mathrm{MHz}\right): \delta=159.17,140.08,130.70,129.20$, 122.97, 113.81, 77.32, 70.50, 70.37, 55.28, 39.39, 29.91, 22.34, 16.66, $14.17,13.97$

HRMS (ES+): $m / z$ [315.1936] $]^{+}$calcd for $\mathrm{C}_{18} \mathrm{H}_{28} \mathrm{O}_{3} \mathrm{Na}^{+}[\mathrm{M}+\mathrm{Na}]^{+}$; found: 315.1944 .

\section{(2R,E)-2-Hydroxy-5-methylnon-4-en-3-yl Benzoate (20)}

Prepared according to the general benzoylation procedure using $\mathbf{1 8}$ $(0.431 \mathrm{~g}, 1.3 \mathrm{mmol})$. The general procedure for DDQ removal of the PMB was then performed on the crude benzoylation product mixture obtained. Purification by flash chromatography on silica gel gave $\mathbf{2 0}$ $(0.344 \mathrm{~g}, 89 \%)$ as a colorless oil; $R_{f}=0.32$ (4:1 hexanes:EtOAc).

IR (ATR): 3462, 3062, 2956, 2929, 2871, 1714, 1600, 1578, 1450, 1315 , $1266,1111,1068,962,709 \mathrm{~cm}^{-1}$.

${ }^{1} \mathrm{H} \mathrm{NMR}\left(\mathrm{CDCl}_{3}, 500 \mathrm{MHz}\right): \delta=8.05(\mathrm{dd}, J=8.5,1.3 \mathrm{~Hz}, 4 \mathrm{H}), 7.55(\mathrm{t}, J=$ $7.4 \mathrm{~Hz}, 2 \mathrm{H}), 7.44(\mathrm{t}, J=7.7 \mathrm{~Hz}, 4 \mathrm{H}), 5.66(\mathrm{dd}, J=9.2,4.4 \mathrm{~Hz}, 1 \mathrm{H}), 5.57$ $(\mathrm{dd}, J=9.5,7.2 \mathrm{~Hz}, 1 \mathrm{H}), 5.32(\mathrm{dq}, J=9.2,1.3 \mathrm{~Hz}, 1 \mathrm{H}), 5.20$ (dq, $J=9.5$, $1.3 \mathrm{~Hz}, 1 \mathrm{H}$ ), 4.03 (qd, $J=6.3,4.2 \mathrm{~Hz}, 1 \mathrm{H}$ ), 3.97 (pent, $J=6.6 \mathrm{~Hz}, 1 \mathrm{H}$ ), $2.06(\mathrm{t}, J=7.6 \mathrm{~Hz}, 2 \mathrm{H}), 2.04(\mathrm{t}, J=7.3 \mathrm{~Hz}, 2 \mathrm{H}), 1.84(\mathrm{~d}, J=1.4 \mathrm{~Hz}, 3 \mathrm{H})$, $1.81(\mathrm{~d}, J=1.4 \mathrm{~Hz}, 3 \mathrm{H}), 1.40(\mathrm{~m}, 4 \mathrm{H}), 1.29$ (pent, $J=7.4 \mathrm{~Hz}, 4 \mathrm{H}), 1.24$ $(\mathrm{d}, J=6.5 \mathrm{~Hz}, 3 \mathrm{H}), 1.22(\mathrm{~d}, J=6.5 \mathrm{~Hz}, 3 \mathrm{H}), 0.89(\mathrm{t}, J=7.3 \mathrm{~Hz}, 3 \mathrm{H}), 0.88$ $(\mathrm{t}, J=7.3 \mathrm{~Hz}, 3 \mathrm{H})$.
${ }^{13} \mathrm{C}$ NMR $\left(\mathrm{CDCl}_{3}, 126 \mathrm{MHz}\right): \delta=166.05,165.96,144.48,144.07$, $132.97,132.95,130.44,130.42,129.64,129.63,128.37,119.36$, $118.42,76.80,75.83,69.74,69.54,39.50,39.46,29.86,29.80,22.32$, $22.30,18.73,18.12,17.20,17.09,13.94$.

HRMS (ES+): $m / z[319.1310]^{+}$calcd for $\mathrm{C}_{19} \mathrm{H}_{20} \mathrm{O}_{3} \mathrm{Na}^{+}[\mathrm{M}+\mathrm{Na}]^{+}$; found: 319.1314 .

\section{(2R,5R,E)-5-Phenylhex-3-en-2-ol (21)}

To a dry Schlenk tube containing a solution of $\mathrm{SmI}_{2}$ in THF $(0.1 \mathrm{M}, 7.0$ $\mathrm{mL}, 7$ equiv) was added degassed nano-pure $\mathrm{H}_{2} \mathrm{O}$ ( $2.5 \mathrm{~mL}, 1400$ equiv) turning the solution to a deep red color. The solution was stirred for 5 min before compound 19 ( $0.030 \mathrm{~g}, 0.10 \mathrm{mmol})$ was then added. After $30 \mathrm{~min}$, the reaction was quenched with aq $\mathrm{NaHCO}_{3}(15 \mathrm{~mL})$ and extracted with EtOAc $(3 \times 15 \mathrm{~mL})$. The combined organic extracts were dried $\left(\mathrm{MgSO}_{4}\right)$, and concentrated in vacuo. Purification by flash chromatography on silica gel gave $21(0.011 \mathrm{~g}, 60 \%)$ as a colorless oil (d.r. = 84:16); $R_{f}=0.30$ ( $4: 1$ hexanes:EtOAc).

IR (ATR): 3462, 3062, 2956, 2929, 2871, 1714, 1600, 1578, 1450, 1315 , $1266,1111,1068,962,709 \mathrm{~cm}^{-1}$.

\section{Major Diastereomer}

${ }^{1} \mathrm{H} \mathrm{NMR}\left(\mathrm{CDCl}_{3}, 500 \mathrm{MHz}\right): \delta=7.30(\mathrm{t}, J=7.6 \mathrm{~Hz}, 2 \mathrm{H}), 7.22-7.18(\mathrm{~m}, 3$ H), 5.82 (ddd, $J=15.4,6.7,1.1 \mathrm{~Hz}, 1 \mathrm{H}$ ), 5.56 (ddd, $J=15.5,6.6,1.4 \mathrm{~Hz}$, $1 \mathrm{H}$ ), 4.30 (pent, $J=6.4 \mathrm{~Hz}, 1 \mathrm{H}$ ), 3.46 (pent, $J=7.0,6.4 \mathrm{~Hz}, 1 \mathrm{H}$ ), 1.36 $(\mathrm{d}, J=7.0 \mathrm{~Hz}, 3 \mathrm{H}), 1.28(\mathrm{~d}, J=6.4 \mathrm{~Hz}, 3 \mathrm{H})$.

${ }^{13} \mathrm{C}$ NMR $\left(\mathrm{CDCl}_{3}, 126 \mathrm{MHz}\right): \delta=145.56,135.41,132.87,128.44$, $127.16,126.16,68.87,41.83,23.42,21.17$.

HRMS (ES+): $m / z$ [159.1174] $]^{+}$calcd for $\mathrm{C}_{12} \mathrm{H}_{15}[\mathrm{M}-\mathrm{OH}]^{+}$; found: 159.1175 .

\section{(2R,5S,E)-5-Methylnon-3-en-2-ol (22)}

To a dry Schlenk tube containing a solution of $\mathrm{SmI}_{2}$ in $\operatorname{THF}(0.1 \mathrm{M}, 7.7$ $\mathrm{mL}, 7$ equiv) was added degassed nano-pure $\mathrm{H}_{2} \mathrm{O}(2.75 \mathrm{~mL}, 1400$ equiv) turning the solution to a deep red color. The solution was stirred for $5 \mathrm{~min}$ before compound $\mathbf{2 0}(0.030 \mathrm{~g}, 0.11 \mathrm{mmol})$ was added. After $30 \mathrm{~min}$, the reaction was quenched with aq $\mathrm{NaHCO}_{3}(15 \mathrm{~mL})$ and extracted with EtOAc $(3 \times 15 \mathrm{~mL})$. The combined organic extracts were dried $\left(\mathrm{MgSO}_{4}\right)$, and concentrated in vacuo. Purification by flash chromatography on silica gel gave $22(0.010 \mathrm{~g}, 60 \%)$ as a colorless oil (d.r. $=90: 10) ; R_{f}=0.38$ ( $4: 1$ hexanes:EtOAc).

IR (ATR): 3347, 2958, 2925, 2871, 2857, 1606, 1457, 1371, 1258, 1150, $1123,1060,969,730 \mathrm{~cm}^{-1}$

\section{Major Diastereomer}

${ }^{1} \mathrm{H} \mathrm{NMR}\left(\mathrm{CDCl}_{3}, 500 \mathrm{MHz}\right): \delta=5.54(\mathrm{dd}, J=15.4,6.9 \mathrm{~Hz}, 1 \mathrm{H}), 5.48(\mathrm{dd}$, $J=15.4,6.0 \mathrm{~Hz}, 1 \mathrm{H}$ ), 4.28 (pent, $J=6.3 \mathrm{~Hz}, 1 \mathrm{H}$ ), 2.11 (pent, $J=6.6 \mathrm{~Hz}$, $1 \mathrm{H}), 1.29(\mathrm{~m}, 6 \mathrm{H}), 1.28(\mathrm{~d}, J=6.3 \mathrm{~Hz}, 3 \mathrm{H}), 0.99(\mathrm{~d}, J=6.8 \mathrm{~Hz}, 3 \mathrm{H})$, $0.90(\mathrm{t}, J=7.0 \mathrm{~Hz}, 3 \mathrm{H})$.

${ }^{13} \mathrm{C}$ NMR $\left(\mathrm{CDCl}_{3}, 126 \mathrm{MHz}\right): \delta=136.99,132.24,69.03,36.56,36.14$, 29.48, 23.49, 22.79, 20.40, 14.08 .

HRMS (ES+): $m / z$ [139.1487] $]^{+}$calcd for $\mathrm{C}_{10} \mathrm{H}_{19}[\mathrm{M}-\mathrm{OH}]^{+}$; found: 139.1482 .

(5S,E)-7-[(4-Methoxybenzyl)oxy]-5-methyl-2-phenylhept-2-en-4ol (24)

Prepared according to the general procedure for $\mathrm{Zr}$-catalyzed carboaluminations using (2S)-4-\{[(1,1-dimethylethyl)dimethylsilyl $]$ oxy $\}$ -2-methylbutanal $\left(\mathbf{2 3} ;{ }^{36} 0.29 \mathrm{~g}, 1.3 \mathrm{mmol}\right)$. Purification by flash 
chromatography on silica gel gave $\mathbf{2 4}(0.288 \mathrm{~g}, 65 \%)$ as a colorless oil (d.r. $=60: 40) ; R_{f}=0.65$ (1:1 hexanes:EtOAc).

IR (ATR): 3396, 3102, 3080, 3056, 3028, 2931, 2863, 1611, 1585, 1511 , 1493, 1444, 1364, 1301, 1245, 1081, 1032, 909, 820, 757, 731, 696 $\mathrm{cm}^{-1}$.

${ }^{1} \mathrm{H} \mathrm{NMR}\left(\mathrm{CDCl}_{3}, 500 \mathrm{MHz}\right): \delta=7.42-7.38(\mathrm{~m}, 4 \mathrm{H}), 7.32(\mathrm{t}, 7.32, J=7.3$ $\mathrm{Hz}, 4 \mathrm{H}), 7.27$ (dd, $J=5.6,2.1 \mathrm{~Hz}, 2 \mathrm{H}), 7.26(\mathrm{dd}, J=7.0,2.0 \mathrm{~Hz}, 2 \mathrm{H}$ ), 7.25 (tt, $J=6.3,1.3 \mathrm{~Hz}, 2 \mathrm{H}), 6.88(\mathrm{~d}, J=8.6 \mathrm{~Hz}, 2 \mathrm{H}), 6.87(\mathrm{~d}, J=8.6 \mathrm{~Hz}$, $2 \mathrm{H}), 5.82(\mathrm{dq}, J=8.7,1.4 \mathrm{~Hz}, 1 \mathrm{H}), 5.77(\mathrm{dq}, J=8.9,1.4 \mathrm{~Hz}, 1 \mathrm{H}), 4.47(\mathrm{~s}$, $2 \mathrm{H}), 4.47(\mathrm{~d}, J=11.5 \mathrm{~Hz}, 1 \mathrm{H}), 4.44(\mathrm{~d}, J=11.5 \mathrm{~Hz}, 1 \mathrm{H}), 4.43(\mathrm{dd}, J=$ 9.1, $4.5 \mathrm{~Hz}, 1 \mathrm{H}), 4.30(\mathrm{dd}, J=8.8,6.9 \mathrm{~Hz}, 1 \mathrm{H}), 3.81(\mathrm{~s}, 3 \mathrm{H}), 3.80(\mathrm{~s}, 3$ H), 3.63-3.56 (m, $2 \mathrm{H}), 3.54-3.49$ ( $\mathrm{m}, 2 \mathrm{H}), 2.63$ (br, OH), 2.40 (br, OH), $2.08(\mathrm{~d}, J=1.4 \mathrm{~Hz}, 3 \mathrm{H}), 2.07$ (d, $J=1.4 \mathrm{~Hz}, 3 \mathrm{H}), 1.92-1.81(\mathrm{~m}, 4 \mathrm{H})$, 1.64 (sept, $J=6.7 \mathrm{~Hz}, 1 \mathrm{H}$ ), 1.50 (sept, $J=6.6 \mathrm{~Hz}, 1 \mathrm{H}$ ), 0.98 (d, $J=6.5$ $\mathrm{Hz}, 3 \mathrm{H}), 0.93$ (d, $J=6.8 \mathrm{~Hz}, 3 \mathrm{H})$.

${ }^{13} \mathrm{C}$ NMR $\left(\mathrm{CDCl}_{3}, 126 \mathrm{MHz}\right): \delta=159.23,143.31,137.56,137.26$, $130.20,129.55,129.39,129.37,129.19,128.20,127.14,125.90$, 113.84, 113.83, 72.78, 72.77 72.71, 72.20, 68.36, 68.03, 55.28, 37.80, 37.56, 32.96, 32.77, 16.61, 16.51, 16.00, 15.21.

HRMS (ES+): $m / z$ [363.1936 $]^{+}$calcd for $\mathrm{C}_{22} \mathrm{H}_{28} \mathrm{O}_{3} \mathrm{Na}^{+}[\mathrm{M}+\mathrm{Na}]^{+}$; found: 363.1939.

(5S,E)-7-Hydroxy-5-methyl-2-phenylhept-2-en-4-yl Benzoate (25) Prepared according to the general benzoylation procedure using $\mathbf{2 4}$ $(0.288 \mathrm{~g}, 0.85 \mathrm{mmol})$. The general procedure for DDQ removal of the PMB was then performed on the crude benzoylation product mixture obtained. Purification by flash chromatography on silica gel gave $\mathbf{2 5}$ $\left(0.238 \mathrm{~g}, 86 \%\right.$ over two steps) as a colorless oil; $R_{f}=0.15$ (4:1 hexanes: EtOAc).

IR (ATR): 3047, 3059, 3031, 2967, 2931, 2877 1713, 1600, 1583, 1450, $1314,1266,1175,1108,1068,909,848,731 \mathrm{~cm}^{-1}$.

\section{Major Diastereomer}

${ }^{1} \mathrm{H} \mathrm{NMR}\left(\mathrm{CDCl}_{3}, 500 \mathrm{MHz}\right): \delta=7.40(\mathrm{dt}, J=8.4,1.5 \mathrm{~Hz}, 4 \mathrm{H}), 7.33(\mathrm{tt}, J=$ $8.3,1.0 \mathrm{~Hz}, 4 \mathrm{H}), 7.27(\mathrm{tt}, J=4.1,1.3 \mathrm{~Hz}, 2 \mathrm{H}), 7.24(\mathrm{~d}, J=8.5 \mathrm{~Hz}, 4 \mathrm{H})$, $6.87(\mathrm{~d}, J=8.6 \mathrm{~Hz}, 2 \mathrm{H}), 6.85(\mathrm{~d}, J=8.6 \mathrm{~Hz}, 2 \mathrm{H}), 5.80(\mathrm{dq}, J=8.9,1.5 \mathrm{~Hz}$, $1 \mathrm{H}), 5.78(\mathrm{dq}, J=9.0,1.4 \mathrm{~Hz}, 1 \mathrm{H}), 4.44(\mathrm{~s}, 2 \mathrm{H}), 4.43$ (s, $2 \mathrm{H}), 4.38$ (dd, $J=8.9,5.7 \mathrm{~Hz}, 1 \mathrm{H}), 4.35(\mathrm{dd}, J=8.9,6.5 \mathrm{~Hz}, 1 \mathrm{H}), 3.80(\mathrm{~s}, 3 \mathrm{H}), 3.79(\mathrm{~s}$, $3 \mathrm{H}), 3.48-3.42(\mathrm{~m}, 4 \mathrm{H}), 2.10(\mathrm{~d}, J=1.4 \mathrm{~Hz}, 3 \mathrm{H}), 2.09(\mathrm{~d}, J=1.4 \mathrm{~Hz}, 3$ H), $1.80-1.59(\mathrm{~m} 10 \mathrm{H}), 1.00(\mathrm{~d}, J=6.7 \mathrm{~Hz}, 3 \mathrm{H}), 0.93(\mathrm{~d}, J=6.7 \mathrm{~Hz}, 3$ $\mathrm{H})$.

${ }^{13} \mathrm{C}$ NMR $\left(\mathrm{CDCl}_{3}, 126 \mathrm{MHz}\right): \delta=166.33,166.30,143.26,143.23$, $140.64,140.32,133.21,130.95,129.96,129.95,128.71,128.59$, $127.82,127.80,126.33,124.91,124.56,75.94,75.78,61.33,61.24$, 35.77, 35.74, 35.20, 34.97, 17.27, 17.18, 15.78, 15.61.

HRMS (ES+): $m / z$ [347.1623 $]^{+}$calcd for $\mathrm{C}_{21} \mathrm{H}_{24} \mathrm{O}_{3} \mathrm{Na}^{+}[\mathrm{M}+\mathrm{Na}]^{+}$; found: 347.1622 .

\section{(5S,E)-8-[(4-Methoxybenzyl)oxy]-5-methyl-2-phenyloct-2-en-4-ol} (29)

To a Schlenk flask containing $\mathrm{Et}_{2} \mathrm{O}(5.2 \mathrm{~mL})$ and $t$-BuLi $(1.7 \mathrm{M}, 0.917$ $\mathrm{mL}, 1.56 \mathrm{~mL})$ at $-78{ }^{\circ} \mathrm{C}$ was added vinyl iodide $27^{30}(0.190 \mathrm{~g}, 0.78$ $\mathrm{mmol}$ ) dropwise. The solution was stirred for $10 \mathrm{~min}$ at $-78^{\circ} \mathrm{C}$ before adding (2S)-5-[(4-methoxyphenyl)methoxy]-2-methylpentanal $\left(\mathbf{2 8} ;{ }^{37} 0.123 \mathrm{~g}, 0.520 \mathrm{mmol}\right)$ dropwise, and the reaction mixture was stirred for $1 \mathrm{~h}$ at $-78{ }^{\circ} \mathrm{C}$. The reaction was quenched with aq $\mathrm{NH}_{4} \mathrm{Cl}$ $(30 \mathrm{~mL})$ and extracted with EtOAc $(3 \times 20 \mathrm{~mL})$. The combined organic extracts were dried $\left(\mathrm{MgSO}_{4}\right)$, and concentrated in vacuo. Purification by flash chromatography over silica gel gave $29(0.097 \mathrm{~g}, 58 \%)$ as a colorless oil (d.r. $=\sim 50: 50) ; R_{f}=0.64$ (1:1 hexanes:EtOAc).

IR (ATR): 3412, 3080, 3056, 3031, 2931, 2856, 1611, 1585, 1511, 1493 , $1444,1362,1301,1245,1172,1092,1032,821,758,735,696 \mathrm{~cm}^{-1}$.

${ }^{1} \mathrm{H} \mathrm{NMR}\left(\mathrm{CDCl}_{3}, 500 \mathrm{MHz}\right): \delta=7.40(\mathrm{dt}, J=8.4,1.5 \mathrm{~Hz}, 4 \mathrm{H}), 7.33(\mathrm{tt}, J=$ 8.3, $1.0 \mathrm{~Hz}, 4 \mathrm{H}), 7.27(\mathrm{tt}, J=4.1,1.3 \mathrm{~Hz}, 2 \mathrm{H}), 7.24(\mathrm{~d}, J=8.5 \mathrm{~Hz}, 4 \mathrm{H})$, $6.87(\mathrm{~d}, J=8.6 \mathrm{~Hz}, 2 \mathrm{H}), 6.85(\mathrm{~d}, J=8.6 \mathrm{~Hz}, 2 \mathrm{H}), 5.80(\mathrm{dq}, J=8.9,1.5 \mathrm{~Hz}$, $1 \mathrm{H}), 5.78(\mathrm{dq}, J=9.0,1.4 \mathrm{~Hz}, 1 \mathrm{H}), 4.44(\mathrm{~s}, 2 \mathrm{H}), 4.43(\mathrm{~s}, 2 \mathrm{H}), 4.38(\mathrm{dd}$, $J=8.9,5.7 \mathrm{~Hz}, 1 \mathrm{H}), 4.35(\mathrm{dd}, J=8.9,6.5 \mathrm{~Hz}, 1 \mathrm{H}), 3.80(\mathrm{~s}, 3 \mathrm{H}), 3.79(\mathrm{~s}$, $3 \mathrm{H}), 3.48-3.42(\mathrm{~m}, 4 \mathrm{H}), 2.10$ (d, $J=1.4 \mathrm{~Hz}, 3 \mathrm{H}), 2.09$ (d, $J=1.4 \mathrm{~Hz}, 3$ H), $1.80-1.59(\mathrm{~m} 10 \mathrm{H}), 1.00(\mathrm{~d}, J=6.7 \mathrm{~Hz}, 3 \mathrm{H}), 0.93(\mathrm{~d}, J=6.7 \mathrm{~Hz}, 3$ $\mathrm{H})$.

${ }^{13} \mathrm{C}$ NMR $\left(\mathrm{CDCl}_{3}, 126 \mathrm{MHz}\right): \delta=159.09,143.17,138.01,137.65$, $130.68,129.38,129.24,129.22,128.98,128.24,127.25,127.22$, $125.86,113.75,72.67,72.65,72.57,72.55,70.38,70.32,55.27,39.51$, 39.44, 28.96, 28.93, 27.53, 27.21, 16.59, 16.50, 14.97, 14.95 .

HRMS (ES+): $m / z$ [377.2093 $]^{+}$calcd for $\mathrm{C}_{23} \mathrm{H}_{30} \mathrm{O}_{3} \mathrm{Na}^{+}[\mathrm{M}+\mathrm{Na}]^{+}$; found: 377.2094.

\section{(5S,E)-8-Hydroxy-5-methyl-2-phenyloct-2-en-4-yl Benzoate (30)}

Prepared according to the general benzoylation procedure using $\mathbf{2 9}$ $(0.097 \mathrm{~g}, 0.27 \mathrm{mmol})$. The general procedure for DDQ removal of the $\mathrm{PMB}$ was then performed on the crude benzoylation product mixture obtained. Purification by flash chromatography on silica gel gave 30 (0.065 g, 71\%) as a colorless oil; $R_{f}=0.13$ ( $4: 1$ hexanes:EtOAc).

IR (ATR): 3400, 3059, 3031, 2931, 2876, 1712, 1600, 1583, 1493, 1450, $1380,1314,1266,1175,1107,1068,1025,908,731 \mathrm{~cm}^{-1}$.

${ }^{1} \mathrm{H} \mathrm{NMR}\left(\mathrm{CDCl}_{3}, 500 \mathrm{MHz}\right): \delta=8.06(\mathrm{dt}, J=8.4,1.0 \mathrm{~Hz}, 4 \mathrm{H}), 7.55$ (tq, $J=6.9,1.3 \mathrm{~Hz}, 2 \mathrm{H}), 7.44(\mathrm{t}, J=7.6 \mathrm{~Hz}, 4 \mathrm{H}), 7.40(\mathrm{dt}, J=8.5,1.5 \mathrm{~Hz}, 4$ $\mathrm{H}), 7.31(\mathrm{tt}, J=7.38,1.0 \mathrm{~Hz}, 4 \mathrm{H}), 7.26(\mathrm{tq}, J=7.2,1.3 \mathrm{~Hz}, 2 \mathrm{H}), 5.82(\mathrm{~m}$, $4 \mathrm{H}), 3.67(\mathrm{tt}, J=6.5,2.0 \mathrm{~Hz}, 4 \mathrm{H}), 2.24(\mathrm{~d}, J=1.0 \mathrm{~Hz}, 3 \mathrm{H}), 2.23(\mathrm{~s}, 3 \mathrm{H})$, $2.04(\mathrm{~m}, 1 \mathrm{H}), 1.97(\mathrm{~m}, 1 \mathrm{H}), 1.77-1.54(\mathrm{~m}, 8 \mathrm{H}), 1.12(\mathrm{~d}, J=6.9 \mathrm{~Hz}, 3$ $\mathrm{H}), 1.09(\mathrm{~d}, J=6.8 \mathrm{~Hz}, 3 \mathrm{H})$.

${ }^{13} \mathrm{C}$ NMR $\left(\mathrm{CDCl}_{3}, 126 \mathrm{MHz}\right): \delta=165.97,165.94,142.93,142.89$, $140.14,139.65,132.79,130.65,129.57,128.31,128.20,127.40$, $127.38,125.95,125.94,124.83,124.27,75.53,75.45,63.07,63.06$, 38.05, 37.56, 30.45, 30.17, 28.60, 28.56, 16.87, 16.78, 15.30, 15.05 .

HRMS (ES+): $m / z$ [361.1780 $]^{+}$calcd for $\mathrm{C}_{22} \mathrm{H}_{26} \mathrm{O}_{3} \mathrm{Na}^{+}[\mathrm{M}+\mathrm{Na}]^{+}$; found: 361.1780 .

\section{(6S,E)-6-[(tert-Butyldimethylsilyl)oxy]-2-phenylhept-2-en-4-ol}

(35)

Prepared according to the general procedure for $\mathrm{Zr}$-catalyzed carboaluminations using (S)-3-[(tert-butyldimethylsilyl)oxy]butanal $(32 ; 380.231 \mathrm{~g}, 1.15 \mathrm{mmol})$. Purification by flash chromatography on silica gel gave $35(0.234 \mathrm{~g}, 63 \%)$ as a colorless oil (d.r. = 54:46); $R_{f}=$ 0.52 (4:1 hexanes:EtOAc).

IR (ATR): 3413, 3081, 3057, 3027, 2955, 2928, 2855, 1612, 1512, 1494, $1462,1374,1248,1143,1077,1001,834,807,774,756,731,695 \mathrm{~cm}^{-1}$. ${ }^{1} \mathrm{H} \mathrm{NMR}\left(\mathrm{CDCl}_{3}, 500 \mathrm{MHz}\right): \delta=7.41(\mathrm{~d}, J=7.1 \mathrm{~Hz}, 4 \mathrm{H}), 7.31(\mathrm{t}, J=7.4$ $\mathrm{Hz}, 4 \mathrm{H}), 7.24$ (t, J = 7.2 Hz $2 \mathrm{H}$ ), 5.83 (dq, $J=8.6,1.3 \mathrm{~Hz}, 1 \mathrm{H}$ ), 5.79 (dq, $J=8.2,1.4 \mathrm{~Hz}, 2 \mathrm{H}), 4.90(\mathrm{dt}, J=8.9,3.1 \mathrm{~Hz}, 1 \mathrm{H}), 4.72(\mathrm{dt}, J=8.9,3.1$ $\mathrm{Hz}, 1 \mathrm{H}$ ), 4.23 (pentd, $J=6.2,3.6 \mathrm{~Hz}, 1 \mathrm{H}$ ), 4.16 (dtd, $J=12.2,6.1,3.8$ $\mathrm{Hz}, 1 \mathrm{H}$ ), 3.35 (br, OH), 3.13 (br, OH), 2.10 (d, $J=1.4 \mathrm{~Hz}, 3 \mathrm{H}), 2.10$ (d, $J=1.4 \mathrm{~Hz}, 3 \mathrm{H}$ ), 1.83 (ddd, $J=9.1,7.0,3.4 \mathrm{~Hz}, 1 \mathrm{H}$ ), 1.80 (ddd, $J=9.2$, 5.2, $1.6 \mathrm{~Hz}, 1 \mathrm{H}$ ), 1.63 (dddd, $J=14.3,6.91,3.8,3.2 \mathrm{~Hz}, 1 \mathrm{H}$ ), 1.60 (dddd, $J=14.3,8.7,6.2,2.6 \mathrm{~Hz}, 1 \mathrm{H}), 1.28(\mathrm{~d}, J=6.3 \mathrm{~Hz}, 3 \mathrm{H}), 1.23(\mathrm{~d}, J=$ $6.1 \mathrm{~Hz}, 3 \mathrm{H}), 0.93(\mathrm{~s}, 18 \mathrm{H}), 0.14(\mathrm{~d}, J=9.1 \mathrm{~Hz}, 6 \mathrm{H}), 0.12(\mathrm{~d}, J=8.1 \mathrm{~Hz}, 6$ $\mathrm{H})$. 
${ }^{13} \mathrm{C}$ NMR $\left(\mathrm{CDCl}_{3}, 126 \mathrm{MHz}\right): \delta=143.24,143.21,136.54,135.96$, $131.13,130.86,128.33,127.25,127.22,125.99,125.94,69.63,68.69$, $67.20,66.06,46.31,45.03,25.97,24.71,23.29,18.11,18.07,16.52$, $16.28,-3.66,-4.26,-4.65,-4.83$.

HRMS (ES+): $m / z[343.2069]^{+}$calcd for $\mathrm{C}_{19} \mathrm{H}_{32} \mathrm{O}_{2} \mathrm{SiNa}^{+}[\mathrm{M}+\mathrm{Na}]^{+}$; found: 343.2065 .

\section{(6S,E)-6-Hydroxy-2-phenylhept-2-en-4-yl Benzoate (38a)}

Prepared according to the general benzoylation procedure using 35 $(0.234 \mathrm{~g}, 0.729 \mathrm{mmol})$. The crude product mixture was placed into a Teflon reaction vessel containing THF $(7.3 \mathrm{~mL})$, cooled to $0{ }^{\circ} \mathrm{C}$, and treated with HF.pyr (70\% HF, $0.400 \mathrm{~mL}, 12.064 \mathrm{mmol}$ ) and left to sit for $18 \mathrm{~h}$ at $4{ }^{\circ} \mathrm{C}$ without stirring. The reaction was quenched with aq $\mathrm{NaHCO}_{3}$ and extracted with EtOAc $(3 \times 10 \mathrm{~mL})$. The combined organic extracts were dried $\left(\mathrm{MgSO}_{4}\right)$, and concentrated in vacuo. Purification by flash chromatography over silica gel gave 38a $(0.194 \mathrm{~g}$, $86 \%$ over two steps) as a colorless oil; $R_{f}\left(\right.$ diastereomer $\left._{\alpha}\right)=0.24 ; R_{f}$ (diastereo$\left.\operatorname{mer}_{\beta}\right)=0.18(4: 1$ hexanes:EtOAc).

IR (ATR): 3428, 3060, 3032, 1713, 1600, 1584, 1450, 1266, 1108, 1068 , $1025,934,847,731 \mathrm{~cm}^{-1}$

${ }^{1} \mathrm{H} \mathrm{NMR}\left(\mathrm{CDCl}_{3}, 500 \mathrm{MHz}\right): \delta=8.06(\mathrm{t}, J=9.2 \mathrm{~Hz}, 4 \mathrm{H}), 7.58(\mathrm{tt}, J=7.4$, $1.2 \mathrm{~Hz}, 1 \mathrm{H}), 7.55(\mathrm{tt}, J=7.4,1.2 \mathrm{~Hz}, 1 \mathrm{H}), 7.45(\mathrm{t}, J=8.0 \mathrm{~Hz}, 4 \mathrm{H}), 7.41$ $(\mathrm{d}, J=7.51 \mathrm{~Hz}, 4 \mathrm{H}), 7.33(\mathrm{t}, J=7.2 \mathrm{~Hz}, 2 \mathrm{H}), 7.32(\mathrm{t}, J=7.5 \mathrm{~Hz}, 2 \mathrm{H})$, $7.27(\mathrm{tt}, J=5.88,1.3 \mathrm{~Hz}, 2 \mathrm{H}), 6.19(\mathrm{dd}, J=8.8,3.5 \mathrm{~Hz}, 1 \mathrm{H}), 6.10(\mathrm{dt}, J=$ $6.90 \mathrm{~Hz}, 1 \mathrm{H}), 5.90(\mathrm{dq}, J=8.7,1.4 \mathrm{~Hz}, 1 \mathrm{H}), 5.80(\mathrm{dq}, J=9.2,1.2 \mathrm{~Hz}, 1$ $\mathrm{H}), 3.97(\mathrm{qd}, J=6.2,1.4 \mathrm{~Hz}, 1 \mathrm{H}), 3.86(\mathrm{qd}, J=6.2,2.6 \mathrm{~Hz}, 1 \mathrm{H}), 2.26(\mathrm{~d}$, $J=1.2 \mathrm{~Hz}, 3 \mathrm{H}), 2.19(\mathrm{t}, J=1.2 \mathrm{~Hz}, 3 \mathrm{H}), 2.13$ (ddd, $J=14.0,8.3,6.6 \mathrm{~Hz}, 1$ H), 1.99 (ddd, $J=13.3,10.4,2.6 \mathrm{~Hz}, 1 \mathrm{H}$ ), 1.88 (ddd, $J=11.5,7.3,4.5 \mathrm{~Hz}$, $1 \mathrm{H}), 1.80$ (ddd, $J=13.7,10.0,3.3 \mathrm{~Hz}, 1 \mathrm{H}), 1.29(\mathrm{~d}, J=6.2 \mathrm{~Hz}, 3 \mathrm{H}), 1.24$ (d, $J=6.2 \mathrm{~Hz}, 3 \mathrm{H}$ ).

${ }^{13} \mathrm{C}$ NMR $\left(\mathrm{CDCl}_{3}, 126 \mathrm{MHz}\right): \delta=167.10,165.91,142.66,142.52$, $139.76,139.26,133.20,132.91,130.52,130.02,129.79,129.60$, $128.42,128.36,128.30,128.26,127.59,127.53,125.96,125.93$, 125.87, 125.76, 70.75, 69.88, 65.46, 63.63, 45.08, 44.24, 24.14, 23.08, 16.66.

HRMS (ES+): $m / z[333.1467]^{+}$calcd for $\mathrm{C}_{20} \mathrm{H}_{22} \mathrm{O}_{3} \mathrm{Na}^{+}[\mathrm{M}+\mathrm{Na}]^{+}$; found: 333.1483.

\section{(E)-7-[(tert-Butyldimethylsilyl)oxy]-6-methyl-2-phenylhept-2-en- 4-ol (36)}

Prepared according to the general procedure for $\mathrm{Zr}$-catalyzed carboaluminations using 4-[(tert-butyldimethylsilyl)oxy]-3-methylbutanal (33; $\left.{ }^{39} 0.500 \mathrm{~g}, 2.31 \mathrm{mmol}\right)$. Purification by flash chromatography on silica gel gave $\mathbf{3 6}(0.658 \mathrm{~g}, 85 \%)$ as a colorless oil (d.r. = 54:46); $R_{f}=0.51$ ( $4: 1$ hexanes:EtOAc).

IR (ATR): 3347, 3082, 3058, 3028, 2954, 2927, 2855, 1598, 1495, 1471 , 1462, 1387, 1360, 1250, 1153, 1089, 1028, 1005, 833, 774, 755, 694 $\mathrm{cm}^{-1}$.

${ }^{1} \mathrm{H} \mathrm{NMR}\left(\mathrm{CDCl}_{3}, 500 \mathrm{MHz}\right): \delta=7.43(\mathrm{~d}, J=7.8 \mathrm{~Hz}, 4 \mathrm{H}), 7.33(\mathrm{t}, J=7.4$ $\mathrm{Hz}, 4 \mathrm{H}$ ), 7.26 (tq, $J=7.4,1.3 \mathrm{~Hz}, 2 \mathrm{H}$ ), 5.83 (tq, $J=8.5,1.4 \mathrm{~Hz}, 2 \mathrm{H}), 4.72$ $(\mathrm{td}, J=7.3,6.2 \mathrm{~Hz}, 1 \mathrm{H}), 4.64(\mathrm{td}, J=8.8,3.8 \mathrm{~Hz}, 1 \mathrm{H}), 3.60(\mathrm{dd}, J=10$, $4.7 \mathrm{~Hz}, 1 \mathrm{H}), 3.58$ (dd, 5.2, $9.9 \mathrm{~Hz}, 1 \mathrm{H}), 3.52(\mathrm{dd}, J=9.9,6.7 \mathrm{~Hz}, 1 \mathrm{H})$, $3.48(\mathrm{dd}, J=10.0,7.4 \mathrm{~Hz}, 1 \mathrm{H}), 3.25(\mathrm{br}, \mathrm{OH}), 2.95$ (br, OH), 2.12 (d, $J=$ $1.4 \mathrm{~Hz}, 6 \mathrm{H}), 1.89$ (octet, $J=6.6 \mathrm{~Hz}, 2 \mathrm{H}), 1.72(\mathrm{ddd}, J=14.3,9.2,7.1 \mathrm{~Hz}$, $1 \mathrm{H}$ ), 1.66 (ddd, $J=7.2,6.1,3.6 \mathrm{~Hz}, 2 \mathrm{H}$ ), 1.49 (ddd, $J=14.1,5.9,3.7 \mathrm{~Hz}$, $1 \mathrm{H}), 0.98(\mathrm{~d}, J=6.8 \mathrm{~Hz}, 6 \mathrm{H}), 0.95(\mathrm{~s}, 9 \mathrm{H}), 0.95(\mathrm{~s}, 9 \mathrm{H}), 0.12(\mathrm{~d}, J=2.7$ $\mathrm{Hz}, 6 \mathrm{H}), 0.11(\mathrm{~d}, J=2.2 \mathrm{~Hz}, 6 \mathrm{H})$.

${ }^{13} \mathrm{C}$ NMR $\left(\mathrm{CDCl}_{3}, 126 \mathrm{MHz}\right): \delta=143.07,143.05,136.02,135.69$, 131.58, 131.19, 128.08, 126.97, 126.94, 125.74, 69.01, 68.39, 67.71,
$66.58,43.19,42.31,33.90,32.30,25.85,18.26,18.23,17.76,17.38$, $16.14,-5.46,-5.51,-5.53$.

HRMS (ES+): $m / z$ [357.2226] $]^{+}$calcd for $\mathrm{C}_{20} \mathrm{H}_{34} \mathrm{O}_{2} \mathrm{SiNa}^{+}[\mathrm{M}+\mathrm{Na}]^{+}$; found: 357.2222 .

\section{(E)-7-Hydroxy-6-methyl-2-phenylhept-2-en-4-yl Benzoate (38b)}

Prepared according to the general benzoylation procedure using $\mathbf{3 6}$ $(0.250 \mathrm{~g}, 0.747 \mathrm{mmol})$. The crude product mixture was placed into a Teflon reaction vessel containing THF $(7.4 \mathrm{~mL})$, cooled to $0{ }^{\circ} \mathrm{C}$, and treated with HF.pyr (70\% HF, $0.400 \mathrm{~mL}, 12.064 \mathrm{mmol}$ ) and left to sit for $18 \mathrm{~h}$ at $4{ }^{\circ} \mathrm{C}$ without stirring. The reaction was quenched with aq $\mathrm{NaHCO}_{3}$ and extracted with EtOAc $(3 \times 10 \mathrm{~mL})$. The combined organic extracts were dried $\left(\mathrm{MgSO}_{4}\right)$, and concentrated in vacuo. Purification by flash chromatography on silica gel gave $38 b(0.240 \mathrm{~g}$, $99 \%$ over two steps) as a colorless oil; $R_{f}=0.18$ (4:1 hexanes:EtOAc).

IR (ATR): 3429, 3065, 3032, 2962, 2919, 2877, 1712, 1600, 1583, 1450 , $1314,1267,1108,1069,1025,931,711 \mathrm{~cm}^{-1}$.

${ }^{1} \mathrm{H} \mathrm{NMR}\left(\mathrm{CDCl}_{3}, 500 \mathrm{MHz}\right): \delta=8.05(\mathrm{~d}, 8.0 \mathrm{~Hz}, 4 \mathrm{H}), 7.55(\mathrm{tt}, 7.5,1.1$ $\mathrm{Hz}, 2 \mathrm{H}), 7.44(\mathrm{t}, J=7.8 \mathrm{~Hz}, 4 \mathrm{H}), 7.41(\mathrm{dd}, J=7.6,1.8 \mathrm{~Hz}, 4 \mathrm{H}), 7.32(\mathrm{t}$, $7.2 \mathrm{~Hz}, 4 \mathrm{H}), 7.26(\mathrm{tt}, J=7.3,1.2 \mathrm{~Hz}, 2 \mathrm{H}), 6.06(\mathrm{dt}, J=6.8,9.1 \mathrm{~Hz}, 1 \mathrm{H})$, $6.04(\mathrm{dt}, J=8.7,5.3 \mathrm{~Hz}, 1 \mathrm{H}), 5.81(\mathrm{dq}, J=9.0,1.3 \mathrm{~Hz}, 1 \mathrm{H}), 5.78(\mathrm{dq}, J=$ 9.2, $1.3 \mathrm{~Hz}, 1 \mathrm{H}), 3.57(\mathrm{dd}, J=5.6,1.8 \mathrm{~Hz}, 4 \mathrm{H}), 2.24(\mathrm{~d}, J=1.3 \mathrm{~Hz}, 3 \mathrm{H})$, $2.23(\mathrm{~d}, J=1.3 \mathrm{~Hz}, 3 \mathrm{H}), 1.98(\mathrm{~m}, 2 \mathrm{H}), 1.94-1.78(\mathrm{~m}, 4 \mathrm{H}), 1.05(\mathrm{~d}, J=$ $6.7 \mathrm{~Hz}, 3 \mathrm{H}), 1.04(\mathrm{~d}, J=6.4 \mathrm{~Hz}, 3 \mathrm{H})$.

${ }^{13} \mathrm{C}$ NMR $\left(\mathrm{CDCl}_{3}, 126 \mathrm{MHz}\right): \delta=166.15,166.10,142.72,142.69$, $139.52,138.98,132.89,132.87,130.60,130.53,129.63,129.62$, $128.35,128.26,127.50,127.47,126.59,126.35,125.97,125.96,71.00$, 70.58, 68.16, 68.02, 38.67, 32.47, 32.37, 17.17, 16.83, 16.71, 16.65.

HRMS (ES+): $m / z$ [347.1623 $]^{+}$calcd for $\mathrm{C}_{21} \mathrm{H}_{24} \mathrm{O}_{3} \mathrm{Na}^{+}[\mathrm{M}+\mathrm{Na}]^{+}$; found: 347.1619.

\section{(E)-7-[(4-Methoxybenzyl)oxy]-2-phenyloct-2-en-4-ol (37)}

Prepared according to the general procedure for $\mathrm{Zr}$-catalyzed carboaluminations using 4-[(4-methoxybenzyl)oxy]pentanal $\left(\mathbf{3 4} ; 4^{40}\right.$ $0.300 \mathrm{~g}, 1.3 \mathrm{mmol})$. Purification by flash chromatography on silica gel gave $37(0.254 \mathrm{~g}, 57 \%)$ as a colorless oil (d.r. $=50: 50) ; R_{f}=0.61(4: 1$ hexanes:EtOAc).

IR (ATR): 3395, 3080, 3056, 3030, 2930, 2861, 1611, 1585, 1512, 1493 , $1443,1374,1337,1301,1172,1032,911,821,757,733,696 \mathrm{~cm}^{-1}$.

${ }^{1} \mathrm{H} \mathrm{NMR}\left(\mathrm{CDCl}_{3}, 500 \mathrm{MHz}\right): \delta=7.41(\mathrm{~d}, J=7.8 \mathrm{~Hz}, 4 \mathrm{H}), 7.33(\mathrm{t}, J=7.3$ $\mathrm{Hz}, 4 \mathrm{H}), 7.28$ (dd, $J=8.8,1.5 \mathrm{~Hz}, 4 \mathrm{H}), 7.26(\mathrm{tt}, J=6.5,1.2 \mathrm{~Hz}, 2 \mathrm{H})$, $6.88(\mathrm{dd}, J=8.6,2.0 \mathrm{~Hz}, 4 \mathrm{H}), 5.78(\mathrm{dq}, J=5.4,1.4 \mathrm{~Hz}, 1 \mathrm{H}), 5.77(\mathrm{dq} J=$ $5.4,1.4 \mathrm{~Hz}, 1 \mathrm{H}), 4.54(\mathrm{~d}, J=11.5 \mathrm{~Hz}, 4 \mathrm{H}), 4.40(\mathrm{dd}, J=11.3,2.4 \mathrm{~Hz}, 2$ H), $3.80(\mathrm{~s}, 6 \mathrm{H}), 3.57$ (hex, $5.9 \mathrm{~Hz}, 2 \mathrm{H}), 2.08(\mathrm{~d}, J=1.3 \mathrm{~Hz}, 3 \mathrm{H}), 2.08$ (d, $J=1.3 \mathrm{~Hz}, 3 \mathrm{H}), 1.81-1.56(\mathrm{~m}, 8 \mathrm{H}), 1.22(\mathrm{~d}, J=6.2 \mathrm{~Hz}, 6 \mathrm{H})$.

${ }^{13} \mathrm{C}$ NMR $\left(\mathrm{CDCl}_{3}, 126 \mathrm{MHz}\right): \delta=159.06,142.97,142.95,136.80$, $136.72,130.86,130.84,129.26,129.24,128.18,127.15,125.78$, $113.74,74.44,74.43,70.02,69.98,68.96,68.89,55.22,33.67,33.58$, $32.56,32.51,19.49,19.47,16.29,16.27$.

HRMS (ES+): $m / z$ [377.2093 $]^{+}$calcd for $\mathrm{C}_{23} \mathrm{H}_{30} \mathrm{O}_{3} \mathrm{Na}^{+}[\mathrm{M}+\mathrm{Na}]^{+}$; found: 377.2094

\section{(E)-7-Hydroxy-2-phenyloct-2-en-4-yl Benzoate (38c)}

Prepared according to the general benzoylation procedure using 37 $(0.288 \mathrm{~g}, 0.845 \mathrm{mmol})$. The general procedure for DDQ removal of the $\mathrm{PMB}$ was then performed on the crude benzoylation product mixture obtained. Purification by flash chromatography on silica gel gave $\mathbf{3 8 c}$ $\left(0.180 \mathrm{~g}, 74 \%\right.$ over two steps) as a colorless oil; $R_{f}=0.15$ (4:1 hexanes: EtOAc). 
IR (ATR): 3411, 3059, 3031, 2967, 2927, 2866, 1712, 1601, 1583, 1493 , $1450,1376,1314,1267,1175,1109,1069,1025,710 \mathrm{~cm}^{-1}$.

${ }^{1} \mathrm{H} \mathrm{NMR}\left(\mathrm{CDCl}_{3}, 500 \mathrm{MHz}\right): \delta=8.06(\mathrm{dd}, J=8.5,1.3 \mathrm{~Hz}, 4 \mathrm{H}), 7.55(\mathrm{tt}$, $J=7.4,1.2 \mathrm{~Hz}, 2 \mathrm{H}), 7.44(\mathrm{t}, J=8.0 \mathrm{~Hz}, 4 \mathrm{H}), 7.41(\mathrm{dd}, J=7.6,1.6 \mathrm{~Hz}, 4$ $\mathrm{H}), 7.32(\mathrm{t}, J=7.8 \mathrm{~Hz}, 4 \mathrm{H}), 7.26(\mathrm{tt}, J=7.2,1.4 \mathrm{~Hz}, 2 \mathrm{H}), 5.95(\mathrm{dt}, J=6.9$, $6.7 \mathrm{~Hz}, 1 \mathrm{H}), 5.94(\mathrm{dt}, J=6.9,6.7 \mathrm{~Hz}, 1 \mathrm{H}), 5.81(\mathrm{dq}, J=9.0,1.3 \mathrm{~Hz}, 2 \mathrm{H})$, 3.88 (hex, $J=6.5 \mathrm{~Hz}, 2 \mathrm{H}), 2.22(\mathrm{~d}, J=1.4 \mathrm{~Hz}, 3 \mathrm{H}), 2.21(\mathrm{~d}, J=1.3 \mathrm{~Hz}, 3$ $\mathrm{H}), 2.05$ (qt, $J=6.94,5.49 \mathrm{~Hz}, 1 \mathrm{H}), 1.95(\mathrm{dt}, J=6.8,6.4 \mathrm{~Hz}, 1 \mathrm{H}), 1.93$ $(\mathrm{dt}, J=6.7,6.5 \mathrm{~Hz}, 1 \mathrm{H}), 1.83(\mathrm{qt}, J=6.0,3.9 \mathrm{~Hz}, 1 \mathrm{H}), 1.63-1.54(\mathrm{~m}, 4$ $\mathrm{H}), 1.23(\mathrm{~d}, J=6.3 \mathrm{~Hz}, 6 \mathrm{H})$.

${ }^{13} \mathrm{C}$ NMR $\left(\mathrm{CDCl}_{3}, 126 \mathrm{MHz}\right): \delta=166.08,166.03,142.72,142.70$, $139.39,139.32,133.63,132.86,132.85,130.60,130.17,129.62$, $128.48,128.33,128.25,127.49,127.47,126.16,126.14,125.96,72.28$, 72.15, 67.93, 67.88, 34.70, 34.56, 31.42, 31.31, 23.70, 23.67, 16.71.

HRMS (ES+): $m / z$ [361.1780] $]^{+}$calcd for $\mathrm{C}_{22} \mathrm{H}_{26} \mathrm{O}_{3} \mathrm{Na}^{+}[\mathrm{M}+\mathrm{Na}]^{+}$; found: 361.1780 .

(E)-5-\{[(4-Methoxybenzyl)oxy]methyl\}-6-methyl-2-phenylhept-2en-4-ol (45)

Prepared according to the general procedure for $\mathrm{Zr}$-catalyzed carboaluminations using 2-\{[(4-methoxybenzyl)oxy]methyl\}-3-methylbutanal (40; $\left.{ }^{41} 0.346 \mathrm{~g}, 1.46 \mathrm{mmol}\right)$. Purification by flash chromatography on silica gel gave $45(0.392 \mathrm{~g}, 75 \%)$ as a colorless oil (d.r. = 91:9); $R_{f}=0.34$ ( $4: 1$ hexanes:EtOAc).

IR (ATR): 3456, 3080, 3055, 3028, 2956, 2870, 1611, 1585, 1512, 1443 , 1418, 1366, 1301, 1246, 1173, 1079, 1032, 987, 909, 819, 757, 732, $696 \mathrm{~cm}^{-1}$.

\section{Major Diastereomer}

${ }^{1} \mathrm{H} \mathrm{NMR}\left(\mathrm{CDCl}_{3}, 500 \mathrm{MHz}\right): \delta=7.37(\mathrm{dd}, J=8.6,1.4 \mathrm{~Hz}, 2 \mathrm{H}), 7.32(\mathrm{t}, J=$ $7.2 \mathrm{~Hz}, 2 \mathrm{H}$ ), 7.27 (dd, J= 8.7, 2.1 Hz, $2 \mathrm{H}$ ), 7.25 (tt, J= 7.3, $2.2 \mathrm{~Hz}, 1 \mathrm{H}$ ), $6.89(\mathrm{dt}, J=8.7,2.1 \mathrm{~Hz}, 2 \mathrm{H}), 5.81(\mathrm{dq}, J=8.5,1.3 \mathrm{~Hz}, 1 \mathrm{H}), 4.72(\mathrm{dt}, J=$ 8.6, $5.8 \mathrm{~Hz}, 1 \mathrm{H}), 4.49$ (d, $J=11.5 \mathrm{~Hz}, 1 \mathrm{H}), 4.45(\mathrm{~d}, J=11.5 \mathrm{~Hz}, 1 \mathrm{H})$, 3.81 (s, $3 \mathrm{H}$ ), 3.79 (dd, $J=9.5,3.0 \mathrm{~Hz}, 1 \mathrm{H}$ ), 3.68 (dd, $J=9.5,5.7 \mathrm{~Hz}, 1$ H), $2.06(\mathrm{~d}, J=1.3 \mathrm{~Hz}, 3 \mathrm{H}), 2.04$ (hex, $J=6.8 \mathrm{~Hz}, 1 \mathrm{H}), 1.47$ (qd, $J=6.0$, $3.0 \mathrm{~Hz}, 1 \mathrm{H}), 1.05(\mathrm{~d}, J=6.8 \mathrm{~Hz}, 3 \mathrm{H}), 0.91(\mathrm{~d}, J=6.9 \mathrm{~Hz}, 3 \mathrm{H})$.

${ }^{13} \mathrm{C}$ NMR $\left(\mathrm{CDCl}_{3}, 126 \mathrm{MHz}\right): \delta=159.34,143.20,135.77,131.06$, 129.69, 129.47, 128.13, 126.98, 125.82, 113.86, 73.24, 70.87, 69.43, $55.25,50.01,26.35,21.52,19.25,16.12$.

HRMS (ES+): $m / z$ [377.2093] $]^{+}$calcd for $\mathrm{C}_{23} \mathrm{H}_{30} \mathrm{O}_{3} \mathrm{Na}^{+}[\mathrm{M}+\mathrm{Na}]^{+}$; found: 377.2076.

\section{(E)-5-(Hydroxymethyl)-6-methyl-2-phenylhept-2-en-4-yl Benzo- ate (50a)}

Prepared according to the general benzoylation procedure using $\mathbf{4 5}$ $(0.392 \mathrm{~g}, 1.11 \mathrm{mmol})$. The general procedure for DDQ removal of the PMB was then performed on the crude benzoylation product mixture obtained. Purification by flash chromatography on silica gel gave 50a $\left(0.281 \mathrm{~g}, 75 \%\right.$ over two steps) as a colorless oil; $R_{f}=0.50$ ( $4: 1$ hexanes: EtOAc).

IR (ATR): 3459, 3083, 3060, 3080, 2958, 2930, 2884, 1712, 1600, 1583 , 1493, 1450, 1387, 1314, 1266, 1176, 1109, 1069, 1025, 920, $710 \mathrm{~cm}^{-1}$.

\section{Major Diastereomer}

${ }^{1} \mathrm{H}$ NMR $\left(\mathrm{CDCl}_{3}, 500 \mathrm{MHz}\right): \delta=8.03(\mathrm{dd}, J=8.5,1.3 \mathrm{~Hz}, 2 \mathrm{H}), 7.57(\mathrm{tt}$, $J=7.4,1.3 \mathrm{~Hz}, 1 \mathrm{H}), 7.45$ (tt, $J=7.9,1.5 \mathrm{~Hz}, 2 \mathrm{H}), 7.41$ (dd, $J=7.4,1.5$ $\mathrm{Hz}, 2 \mathrm{H}), 7.32(\mathrm{t}, J=7.1 \mathrm{~Hz}, 2 \mathrm{H}), 7.27(\mathrm{tt}, J=7.3,1.3 \mathrm{~Hz}, 1 \mathrm{H}), 6.19$ (dd, $J=9.3,6.7 \mathrm{~Hz}, 1 \mathrm{H}), 5.90(\mathrm{dq}, J=9.3,1.3 \mathrm{~Hz}, 1 \mathrm{H}), 3.90(\mathrm{dd}, J=4.5,3.7$ $\mathrm{Hz}, 2 \mathrm{H}$ ), 2.26 (d, $J=1.4 \mathrm{~Hz}, 3 \mathrm{H}$ ), 2.00 (hexd, $J=6.9,5.1 \mathrm{~Hz}, 1 \mathrm{H}$ ), 1.79 (dtd, $J=6.7,5.0,4.1 \mathrm{~Hz}, 1 \mathrm{H}), 1.09(\mathrm{~d}, J=6.9 \mathrm{~Hz}, 3 \mathrm{H}), 1.04(\mathrm{~d}, J=6.9 \mathrm{~Hz}$, $3 \mathrm{H})$.

${ }^{13} \mathrm{C}$ NMR $\left(\mathrm{CDCl}_{3}, 126 \mathrm{MHz}\right): \delta=166.06,142.67,139.70,133.07$, 130.29, 129.57, 128.48, 128.26, 127.53, 125.95, 125.46, 73.06, 60.79, $51.30,27.00,21.42,19.50,16.69$.

HRMS (ES+): $m / z$ [361.1780 $]^{+}$calcd for $\mathrm{C}_{22} \mathrm{H}_{26} \mathrm{O}_{3} \mathrm{Na}^{+}[\mathrm{M}+\mathrm{Na}]^{+}$; found: 361.1758 .

\section{(E)-5-\{[(4-Methoxybenzyl)oxy]methyl\}-6,6-dimethyl-2-phenyl- hept-2-en-4-ol (46)}

Prepared according to the general procedure for Zr-catalyzed carboaluminations using 2-\{[(4-methoxybenzyl)oxy]methyl\}-3,3-dimethylbutanal (41; $\left.{ }^{42} 0.387 \mathrm{~g}, 1.54 \mathrm{mmol}\right)$. Purification by flash chromatography on silica gel gave $\mathbf{4 6}(0.175 \mathrm{~g}, 38 \%)$ as a colorless oil (d.r. = 77:23); $R_{f}=0.43$ (4:1 hexanes:EtOAc).

IR (ATR): 3445, 3080, 3056, 3027, 2954, 2868, 1611, 1586, 1512, 1493 , 1464, 1443, 1363, 1301, 1246, 1206, 1075, 1034, 986, 819, 757, 696 $\mathrm{cm}^{-1}$.

\section{Major Diastereomer}

${ }^{1} \mathrm{H} \mathrm{NMR}\left(\mathrm{CDCl}_{3}, 500 \mathrm{MHz}\right): \delta=7.33(\mathrm{dd}, J=8.3,1.7 \mathrm{~Hz}, 2 \mathrm{H}), 7.30(\mathrm{t}, J=$ $7.8 \mathrm{~Hz}, 2 \mathrm{H}), 7.26$ (d, $J=8.7 \mathrm{~Hz}, 2 \mathrm{H}), 7.24(\mathrm{tt}, J=6.0,1.6 \mathrm{~Hz}, 1 \mathrm{H}), 6.87$ $(\mathrm{d}, J=8.6 \mathrm{~Hz}, 2 \mathrm{H}), 5.98(\mathrm{dq}, J=8.3,1.4 \mathrm{~Hz}, 1 \mathrm{H}), 4.89(\mathrm{td}, J=8.3,2.5 \mathrm{~Hz}$, $1 \mathrm{H}), 4.46(\mathrm{~d}, J=11.4 \mathrm{~Hz}, 1 \mathrm{H}), 4.42(\mathrm{~d}, J=11.4 \mathrm{~Hz}, 1 \mathrm{H}), 3.83(\mathrm{dd}, J=$ 4.1, $1.5 \mathrm{~Hz}, 2 \mathrm{H}$ ), 3.80 (s, $3 \mathrm{H}$ ), 3.32 (d, $J=8.3 \mathrm{~Hz}, 1 \mathrm{H}$ ), 2.05 (d, $J=1.3$ $\mathrm{Hz}, 3 \mathrm{H}), 1.42$ (td, $J=4.2,2.6 \mathrm{~Hz}, 1 \mathrm{H}), 1.10(\mathrm{~s}, 9 \mathrm{H})$.

${ }^{13} \mathrm{C}$ NMR $\left(\mathrm{CDCl}_{3}, 126 \mathrm{MHz}\right): \delta=159.26,143.33,133.83,132.89$, $129.81,129.45,128.10,126.85,125.81,113.81,73.19,69.71,68.84$, $55.23,52.86,33.39,29.26,15.95$.

HRMS (ES+): $m / z$ [391.2249] $]^{+}$calcd for $\mathrm{C}_{24} \mathrm{H}_{32} \mathrm{O}_{3} \mathrm{Na}^{+}[\mathrm{M}+\mathrm{Na}]^{+}$; found: 391.2257.

\section{(E)-5-(Hydroxymethyl)-6,6-dimethyl-2-phenylhept-2-en-4-ylBen- zoate (50b)}

Prepared according to the general benzoylation procedure using 46 ( $0.144 \mathrm{~g}, 0.391 \mathrm{mmol})$. The general procedure for DDQ removal of the PMB was then performed on the crude benzoylation product mixture obtained. Purification by flash chromatography on silica gel gave 50b $\left(0.060 \mathrm{~g}, 42 \%\right.$ over two steps) as a colorless oil; $R_{f}=0.35$ (4:1 hexanes: EtOAc).

IR (ATR): 3459, 3059, 3030, 2958, 2873, 1713, 1600, 1583, 1493, 1476, $1450,1367,1269,1175,1110,1040,910,711 \mathrm{~cm}^{-1}$.

\section{Major Diastereomer}

${ }^{1} \mathrm{H} \mathrm{NMR}\left(\mathrm{CDCl}_{3}, 500 \mathrm{MHz}\right): \delta=8.02(\mathrm{dd}, J=8.3,1.3 \mathrm{~Hz}, 2 \mathrm{H}), 7.56(\mathrm{tt}$, $J=7.0,1.3 \mathrm{~Hz}, 1 \mathrm{H}), 7.45$ (tt, $J=7.7,1.5 \mathrm{~Hz}, 2 \mathrm{H}), 7.40$ (dd, $J=7.0,1.5$ $\mathrm{Hz}, 2 \mathrm{H}), 7.30$ (tt, $J=7.1,1.5 \mathrm{~Hz}, 2 \mathrm{H}), 7.24(\mathrm{tt}, J=7.1,1.3 \mathrm{~Hz}, 1 \mathrm{H}), 6.33$ $(\mathrm{dd}, J=9.0,2.2 \mathrm{~Hz}, 1 \mathrm{H}), 6.06(\mathrm{dq}, J=9.0,1.4 \mathrm{~Hz}, 1 \mathrm{H}), 4.18(\mathrm{dd}, J=11.7$, $6.1 \mathrm{~Hz}, 1 \mathrm{H}$ ), 4.08 (dd, $J=11.7,4.1 \mathrm{~Hz}, 1 \mathrm{H}), 2.27$ (d, $J=1.4 \mathrm{~Hz}, 3 \mathrm{H}$ ), 1.72 (ddd, $J=6.2,4.1,2.2 \mathrm{~Hz}, 1 \mathrm{H}), 1.08(\mathrm{~s}, 9 \mathrm{H})$.

${ }^{13} \mathrm{C}$ NMR $\left(\mathrm{CDCl}_{3}, 126 \mathrm{MHz}\right): \delta=165.60,142.74,137.24,132.99$, 130.44, 129.47, 128.47, 128.19, 127.33, 127.10, 125.93, 72.25, 60.94, 55.52, 33.20, 28.82, 16.51 .

HRMS (ES+): $m / z$ [375.1936 $]^{+}$calcd for $\mathrm{C}_{23} \mathrm{H}_{28} \mathrm{O}_{3} \mathrm{Na}^{+}[\mathrm{M}+\mathrm{Na}]^{+}$; found: 375.1937.

(E)-1-[(4-Methoxybenzyl)oxy]-2,5-diphenylhex-4-en-3-ol (47)

Prepared according to the general procedure for $\mathrm{Zr}$-catalyzed carboaluminations using 3-[(4-methoxybenzyl)oxy]-2-phenylpropanal 
(42; 43 (0.113 g, $0.42 \mathrm{mmol})$. Purification by flash chromatography on silica gel gave $47(0.097 \mathrm{~g}, 59 \%)$ as a colorless oil (d.r. $=98: 2) ; R_{f}=0.19$ (4:1 hexanes:EtOAc).

IR (ATR): 3419, 3082, 3059, 3028, 2999, 2915, 2858, 1611, 1585, 1512, 1493, 1452, 1362, 1301, 1246, 1173, 1076, 1030, 908, 819, 730, 697 $\mathrm{cm}^{-1}$.

\section{Major Diastereomer}

${ }^{1} \mathrm{H} \mathrm{NMR}\left(\mathrm{CDCl}_{3}, 500 \mathrm{MHz}\right): \delta=7.35-7.27(\mathrm{~m}, 2 \mathrm{H}), 7.26(\mathrm{~d}, J=8.7 \mathrm{~Hz}, 2$ H), 7.24-7.15 (m, $8 \mathrm{H}), 6.88(\mathrm{~d}, J=8.7 \mathrm{~Hz}, 2 \mathrm{H}), 5.60(\mathrm{dq}, J=8.9,1.4 \mathrm{~Hz}$, $1 \mathrm{H}), 4.88(\mathrm{td}, J=8.6,3.0 \mathrm{~Hz}, 1 \mathrm{H}), 4.55(\mathrm{~d}, J=11.6 \mathrm{~Hz}, 1 \mathrm{H}), 4.51(\mathrm{~d}, J=$ $11.6 \mathrm{~Hz}, 1 \mathrm{H}$ ), 3.99 (dd, $J=9.4,8.3 \mathrm{~Hz}, 1 \mathrm{H}), 3.88(\mathrm{dd}, J=9.4,4.7 \mathrm{~Hz}, 1$ $\mathrm{H}), 3.81(\mathrm{~s}, 3 \mathrm{H}), 3.45(\mathrm{~d}, J=3.1 \mathrm{~Hz}, \mathrm{OH}), 3.14(\mathrm{td}, J=8.1,4.6 \mathrm{~Hz}, 1 \mathrm{H})$, $1.82(\mathrm{~d}, J=1.4 \mathrm{~Hz}, 3 \mathrm{H})$.

${ }^{13} \mathrm{C}$ NMR $\left(\mathrm{CDCl}_{3}, 126 \mathrm{MHz}\right): \delta=159.35,143.37,139.37,137.61$, $129.65,129.44,129.06,128.65,128.30,128.03,126.97,126.89$, $125.90,113.89,73.23,73.11,72.64,55.28,51.75,16.40$.

HRMS (ES+): $m / z$ [411.1936] $]^{+}$calcd for $\mathrm{C}_{26} \mathrm{H}_{28} \mathrm{O}_{3} \mathrm{Na}^{+}[\mathrm{M}+\mathrm{Na}]^{+}$; found: 411.1938.

\section{(E)-1-Hydroxy-2,5-diphenylhex-4-en-3-yl Benzoate (50c)}

Prepared according to the general benzoylation procedure was using $47(0.097 \mathrm{~g}, 0.249 \mathrm{mmol})$. The general procedure for DDQ removal of the PMB was then performed on the crude benzoylation product mixture obtained. Purification by flash chromatography on silica gel gave 50c $(0.065 \mathrm{~g}, 78 \%$ over two steps $)$ as a colorless oil; $R_{f}=0.37(4: 1$ hexanes:EtOAc).

IR (ATR): 3460, 3083, 3060, 3029, 2923, 1713, 1600, 1583, 1511, 1493 , $1450,1315,1266,1109,1068,1025,907,710 \mathrm{~cm}^{-1}$.

\section{Major Diastereomer}

${ }^{1} \mathrm{H} \mathrm{NMR}\left(\mathrm{CDCl}_{3}, 500 \mathrm{MHz}\right): \delta=7.99(\mathrm{dd}, J=8.3,1.2 \mathrm{~Hz}, 2 \mathrm{H}), 7.49(\mathrm{tt}$, 7.0, $1.2 \mathrm{~Hz}, 1 \mathrm{H}), 7.37$ (tt, $J=7.9,1.5 \mathrm{~Hz}, 2 \mathrm{H}), 7.25(\mathrm{dd}, J=4.2,1.0 \mathrm{~Hz}, 4$ H), 7.19-7.16 (m, $2 \mathrm{H}), 7.16-7.12(\mathrm{~m}, 2 \mathrm{H}), 7.12-7.09(\mathrm{~m}, 2 \mathrm{H}), 6.17(\mathrm{t}$, $J=9.0 \mathrm{~Hz}, 1 \mathrm{H}), 5.56(\mathrm{dq}, J=9.5,1.4 \mathrm{~Hz}, 1 \mathrm{H}), 3.97(\mathrm{~d}, J=5.9 \mathrm{~Hz}, 2 \mathrm{H})$, $3.26(\mathrm{dt}, J=8.7,5.9 \mathrm{~Hz}, 1 \mathrm{H}), 1.93(\mathrm{~d}, J=1.3 \mathrm{~Hz}, 3 \mathrm{H})$.

${ }^{13} \mathrm{C}$ NMR $\left(\mathrm{CDCl}_{3}, 126 \mathrm{MHz}\right): \delta=166.14,142.79,140.52,138.28$, $133.10,130.13,129.68,129.05,128.56,128.41,128.10,127.38$, $127.36,125.92,124.40,72.63,63.45,53.03,16.76$.

HRMS (ES+): $m / z$ [395.1623] $]^{+}$calcd for $\mathrm{C}_{25} \mathrm{H}_{24} \mathrm{O}_{3} \mathrm{Na}^{+}[\mathrm{M}+\mathrm{Na}]^{+}$; found: 395.1610 .

\section{(E)-2-Benzyl-1-[(4-methoxybenzyl)oxy]-5-phenylhex-4-en-3-ol (48)}

To a Schlenk flask containing $\mathrm{Et}_{2} \mathrm{O}(3.5 \mathrm{~mL})$ and $t$-BuLi $(1.7 \mathrm{M}, 0.905$ $\mathrm{mL}, 1.54 \mathrm{~mL})$ at $-78{ }^{\circ} \mathrm{C}$ was added vinyl iodide $27^{30}(0.171 \mathrm{~g}, 0.702$ $\mathrm{mmol})$ dropwise. The solution was stirred for $10 \mathrm{~min}$ at $-78{ }^{\circ} \mathrm{C}$ and $2-$ benzyl-3-[(4-methoxybenzyl)oxy]propanal $\left(\mathbf{4 3} ;{ }^{43} \quad 0.100 \mathrm{~g}, \quad 0.351\right.$ $\mathrm{mmol}$ ) was then added dropwise. The reaction mixture was stirred for $1 \mathrm{~h}$ at $-78{ }^{\circ} \mathrm{C}$ before being brought to r.t. for $30 \mathrm{~min}$. The reaction was quenched with aq $\mathrm{NH}_{4} \mathrm{Cl}(30 \mathrm{~mL})$ and extracted with EtOAc $(3 \times$ $20 \mathrm{~mL}$ ). The combined organic extracts were dried $\left(\mathrm{MgSO}_{4}\right)$ and concentrated in vacuo. Purification by flash chromatography over silica gel gave $48(0.051 \mathrm{~g}, 36 \%)$ as a colorless oil (d.r. $=50: 50) ; R_{f}=0.22(4: 1$ hexanes:EtOAc).

IR (ATR): 3430, 3034, 2917, 2849, 1600, 1594, 1493, 1454, 1442, 1382 , $1333,1244,1201,1160,1033,968,919,836,745,687 \mathrm{~cm}^{-1}$.

${ }^{1} \mathrm{H} \mathrm{NMR}\left(\mathrm{CDCl}_{3}, 500 \mathrm{MHz}\right): \delta=7.40-7.33(\mathrm{~m}, 6 \mathrm{H}), 7.33-7.28(\mathrm{~m}, 4 \mathrm{H})$, 7.28-7.24 (m, 8 H), 7.23-7.14 (m, $6 \mathrm{H}), 6.89$ (d, J = 8.7 Hz, 4 H), 5.89 (dq, $J=8.9,1.4 \mathrm{~Hz}, 1 \mathrm{H}), 5.87$ (dq, $J=8.6,1.3 \mathrm{~Hz}, 1 \mathrm{H}$ ), 4.75 (dd, $J=8.9$, $3.8 \mathrm{~Hz}, 1 \mathrm{H}), 4.60$ (t, J = 7.3 Hz, $1 \mathrm{H}), 4.46(\mathrm{~d}, J=11.5 \mathrm{~Hz}, 1 \mathrm{H}), 4.44(\mathrm{~d}$, $J=11.5 \mathrm{~Hz}, 1 \mathrm{H}), 4.41(\mathrm{~d}, J=11.5 \mathrm{~Hz}, 2 \mathrm{H}), 4.36(\mathrm{~d}, J=11.5 \mathrm{~Hz}, 1 \mathrm{H})$, $3.81(\mathrm{~s}, 6 \mathrm{H}), 3.71$ (dd, $J=9.4,3.5 \mathrm{~Hz}, 1 \mathrm{H}), 3.51$ (dd, $J=9.2,6.0 \mathrm{~Hz}, 1$ $\mathrm{H}), 3.48(\mathrm{dd}, J=9.7,4.2 \mathrm{~Hz}, 1 \mathrm{H}), 3.46(\mathrm{dd}, J=9.4,5.2 \mathrm{~Hz}, 1 \mathrm{H}), 3.26(\mathrm{br}$, $\mathrm{OH}), 3.10$ (br, OH), 2.93 (dd, $J=13.7,5.8 \mathrm{~Hz}, 1 \mathrm{H}$ ), 2.83 (dd, $J=13.7,5.3$ $\mathrm{Hz}, 1 \mathrm{H}$ ), 2.70 (dd, $J=9.5,3.5 \mathrm{~Hz}, 1 \mathrm{H}), 2.67$ (dd, $J=9.5,3.9 \mathrm{~Hz}, 1 \mathrm{H}$ ), 2.27 (dtt, $J=9.7,5.8,4.1 \mathrm{~Hz}, 1 \mathrm{H}), 2.08$ (d, $J=1.4 \mathrm{~Hz}, 3 \mathrm{H}$ ), $2.05(\mathrm{~m}, 1$ $\mathrm{H}), 2.02(\mathrm{~d}, J=1.3 \mathrm{~Hz}, 3 \mathrm{H})$.

${ }^{13} \mathrm{C}$ NMR $\left(\mathrm{CDCl}_{3}, 126 \mathrm{MHz}\right): \delta=159.30,159.28,143.17,143.09$, $140.33,140.29,137.46,136.81,130.04,129.86,129.81,129.47$, $129.43,129.10,129.07,128.36,128.32,128.30,128.18,128.15$, $127.15,127.10,125.97,125.92,125.86,125.83,113.82,73.12,73.10$, 71.23, 71.22, 70.48, 70.12, 55.23, 46.39, 46.36, 34.74, 32.93, 16.48, 16.27.

HRMS (ES+): $m / z$ [425.2093] $]^{+}$calcd for $\mathrm{C}_{27} \mathrm{H}_{30} \mathrm{O}_{3} \mathrm{Na}^{+}[\mathrm{M}+\mathrm{Na}]^{+}$; found: 425.2112

\section{(E)-2-Benzyl-1-hydroxy-5-phenylhex-4-en-3-yl Benzoate (50d)} Prepared according to the general benzoylation procedure using $\mathbf{4 8}$ $(0.102 \mathrm{~g}, 0.254 \mathrm{mmol})$. The general procedure for DDQ removal of the PMB was then performed on the crude benzoylation product mixture obtained. Purification by flash chromatography on silica gel gave 50d $\left(0.081 \mathrm{~g}, 82 \%\right.$ over two steps) as a colorless oil; $R_{f}=0.32$ (4:1 hexanes: EtOAc).

IR (ATR): 3467, 3104, 3083, 3061, 3026, 2926, 1713, 1600, 1583, 1493 , $1450,1373,1314,1266,1175,1111,1068,1025,909,758,733,711$ $\mathrm{cm}^{-1}$.

${ }^{1} \mathrm{H} \mathrm{NMR}\left(\mathrm{CDCl}_{3}, 500 \mathrm{MHz}\right): \delta=8.08(\mathrm{dd}, J=8.2,1.2 \mathrm{~Hz}, 2 \mathrm{H}), 8.06(\mathrm{dd}$, $J=8.1,1.2 \mathrm{~Hz}, 2 \mathrm{H}), 7.59(\mathrm{~m}, 1 \mathrm{H}), 7.58(\mathrm{~m}, 1 \mathrm{H}), 7.47(\mathrm{t}, J=7.6 \mathrm{~Hz}, 4 \mathrm{H})$, 7.44-7.40 (m, $2 \mathrm{H}), 7.40-7.32(\mathrm{~m}, 6 \mathrm{H}), 7.32-7.27$ (m, $6 \mathrm{H}), 7.26-7.19$ $(\mathrm{m}, 6 \mathrm{H}), 6.23(\mathrm{dd}, J=8.9,4.8 \mathrm{~Hz}, 1 \mathrm{H}), 6.10(\mathrm{dd}, J=9.4,7.6 \mathrm{~Hz}, 1 \mathrm{H})$, $5.97(\mathrm{dq}, J=6.1,1.3 \mathrm{~Hz}, 1 \mathrm{H}), 5.95(\mathrm{dq}, J=6.5,1.3 \mathrm{~Hz}, 1 \mathrm{H}), 3.71(\mathrm{dd}, J=$ 11.7, $3.5 \mathrm{~Hz}, 1 \mathrm{H}), 3.66$ (dd, $J=11.5,4.6 \mathrm{~Hz}, 1 \mathrm{H}), 3.61-3.55(\mathrm{~m}, 2 \mathrm{H})$, $3.05(\mathrm{dd}, J=14.0,4.9 \mathrm{~Hz}, 1 \mathrm{H}), 2.87(\mathrm{dd}, J=13.6,5.1 \mathrm{~Hz}, 1 \mathrm{H}), 2.80$ (dd, $J=13.6,9.8 \mathrm{~Hz}, 1 \mathrm{H}), 2.69$ (dd, $J=14.0,9.5 \mathrm{~Hz}, 1 \mathrm{H}), 2.39$ (ddq, $J=9.6$, 7.2, $4.8 \mathrm{~Hz}, 1 \mathrm{H}), 2.24(\mathrm{~m}, 1 \mathrm{H}), 2.23(\mathrm{~d}, J=1.4 \mathrm{~Hz}, 3 \mathrm{H}), 2.21(\mathrm{~d}, J=1.4$ $\mathrm{Hz}, 3 \mathrm{H})$.

${ }^{13} \mathrm{C}$ NMR $\left(\mathrm{CDCl}_{3}, 126 \mathrm{MHz}\right): \delta=166.46,166.45,142.66,142.64$, $140.96,139.94,139.92,139.91,133.14,133.11,130.16,130.10$, $129.71,129.69,129.19,128.97,128.53,128.49,128.43,128.29$, $128.26,127.63,127.54,126.18,125.99,125.93,124.47,124.32,72.31$, $61.79,60.15,48.05,47.63,33.17,33.04,16.86,16.72$.

(E)-1-[(R)-2,2-Dimethyl-1,3-dioxolan-4-yl]-3-phenylbut-2-en-1-ol (49)

Prepared according to the general procedure for $\mathrm{Zr}$-catalyzed carboaluminations using $(R)$-2,2-dimethyl-1,3-dioxolane-4-carboxaldehyde (44; $0.500 \mathrm{~g}, 3.8 \mathrm{mmol})$. Purification by flash chromatography on silica gel gave $49(0.586 \mathrm{~g}, 50 \%)$ as a colorless oil (d.r. $=74: 26) ; R_{f}=$ 0.66 ( $1: 1$ hexanes:EtOAc).

IR (ATR): 3450, 3060, 3034, 2990, 2800, 1665, 1601, 1585, 1501, 1453 , $1386,1310,1076,853 \mathrm{~cm}^{-1}$.

\section{Major Diastereomer}

${ }^{1} \mathrm{H} \mathrm{NMR}\left(\mathrm{CDCl}_{3}, 500 \mathrm{MHz}\right): \delta=7.40(\mathrm{dd}, J=8.1,1.5 \mathrm{~Hz}, 2 \mathrm{H}), 7.33(\mathrm{t}, J=$ $7.1 \mathrm{~Hz}, 2 \mathrm{H}), 7.27(\mathrm{tt}, J=7.2,1.4 \mathrm{~Hz}, 1 \mathrm{H}), 5.68(\mathrm{dq}, J=8.5,1.4 \mathrm{~Hz}, 1 \mathrm{H})$, 4.73 (ddd, $J=8.4,3.9,2.9 \mathrm{~Hz}, 1 \mathrm{H}$ ), 4.20 (td, $J=6.9,4.1 \mathrm{~Hz}, 1 \mathrm{H}$ ), 4.02 (dd, $J=8.2,6.6 \mathrm{~Hz}, 1 \mathrm{H}), 4.00(\mathrm{dd}, 8.4,8.2 \mathrm{~Hz}, 1 \mathrm{H}), 2.14(\mathrm{~d}, J=1.3 \mathrm{~Hz}, 3$ H), 1.48 (s, $3 \mathrm{H}), 1.39$ (s, $3 \mathrm{H})$. 
${ }^{13} \mathrm{C}$ NMR $\left(\mathrm{CDCl}_{3}, 126 \mathrm{MHz}\right): \delta=142.57,139.96,128.29,127.55$, 125.93, 125.89, 125.05, 109.28, 78.14, 68.35, 64.70, 26.45, 25.22, 16.78.

HRMS (ES+): $m / z$ [271.1310 $]^{+}$calcd for $\mathrm{C}_{15} \mathrm{H}_{20} \mathrm{O}_{3} \mathrm{Na}^{+}[\mathrm{M}+\mathrm{Na}]^{+}$; found: 271.1316 .

\section{(2R,E)-1,2-Dihydroxy-5-phenylhex-4-en-3-yl Benzoate (50e)}

Prepared according to the general benzoylation procedure using $\mathbf{4 9}$ $(0.45 \mathrm{~g}, 2.13 \mathrm{mmol})$. The crude product was then redissolved in $\mathrm{MeOH}$ $(8.5 \mathrm{~mL})$ and pTSA $(0.404 \mathrm{~g}, 2.128 \mathrm{mmol})$ was added. The reaction mixture was stirred for 5 min before quenching with aq $\mathrm{NaHCO}_{3}(10$ $\mathrm{mL})$ and extracting with $\mathrm{DCM}(3 \times 10 \mathrm{~mL})$. The combined organic extracts were dried $\left(\mathrm{MgSO}_{4}\right)$, and concentrated in vacuo. Purification by flash chromatography on silica gel gave $\mathbf{5 0 e}(0.223 \mathrm{~g}, 83 \%)$ as a colorless oil; $R_{f}=0.25$ ( $1: 1$ hexanes:EtOAc).

IR (ATR): 3389, 3061, 3032, 2926, 2881, 1712, 1600, 1583, 1493, 1450, $1382,1265,1176,1111,1068,1025,907,710 \mathrm{~cm}^{-1}$.

\section{Major Diastereomer}

${ }^{1} \mathrm{H} \mathrm{NMR}\left(\mathrm{CDCl}_{3}, 500 \mathrm{MHz}\right): \delta=8.08(\mathrm{dd}, J=8.0,1.4 \mathrm{~Hz}, 2 \mathrm{H}), 7.59(\mathrm{tt}$, $J=7.4,1.4 \mathrm{~Hz}, 1 \mathrm{H}$ ), 7.47 (t, $J=8.1 \mathrm{~Hz}, 2 \mathrm{H}), 7.45$ (dd, $J=8.5,1.5 \mathrm{~Hz}, 2$ $\mathrm{H}), 7.35(\mathrm{t}, J=7.0 \mathrm{~Hz}, 2 \mathrm{H}), 7.30(\mathrm{tt}, J=7.15,1.4 \mathrm{~Hz}, 1 \mathrm{H}), 5.99(\mathrm{dd}, J=$ 9.3, $5.8 \mathrm{~Hz}, 1 \mathrm{H}), 5.94(\mathrm{dq}, J=9.1,1.3 \mathrm{~Hz}, 1 \mathrm{H}), 4.03(\mathrm{td}, J=5.9,3.2 \mathrm{~Hz}$, $1 \mathrm{H}), 3.85(\mathrm{dd}, J=11.7,3.3 \mathrm{~Hz}, 1 \mathrm{H}), 3.76(\mathrm{dd}, J=11.7,6.1 \mathrm{~Hz}, 1 \mathrm{H}), 2.26$ (d, $J=1.3 \mathrm{~Hz}, 3 \mathrm{H}$ ).

${ }^{13} \mathrm{C}$ NMR $\left(\mathrm{CDCl}_{3}, 126 \mathrm{MHz}\right): \delta=166.22,142.54,142.26,133.30$, 129.75, 128.45, 128.29, 127.80, 125.97, 121.93, 73.59, 72.10, 62.78, 16.95.

HRMS (ES+): $m / z$ [335.1259] $]^{+}$calcd for $\mathrm{C}_{19} \mathrm{H}_{20} \mathrm{O}_{4} \mathrm{Na}^{+}[\mathrm{M}+\mathrm{Na}]^{+}$; found: 335.1265 .

\section{2-Isopropyl-5-phenylhex-3-en-1-ol (51a)}

Prepared according to the general procedure for $\mathrm{SmI}_{2}\left(\mathrm{H}_{2} \mathrm{O}\right)_{n}$ reductions using 50a $(0.050 \mathrm{~g}, 0.147 \mathrm{mmol})$. Purification by flash chromatography on silica gel gave $\mathbf{5 1 a}(0.026 \mathrm{~g}, 80 \%)$ as a colorless oil (d.r. = 83:17); $R_{f}=0.37$ ( $4: 1$ hexanes:EtOAc).

IR (ATR): 3352, 3056, 2959, 2926, 2870, 1600, 1580, 1492, 1451, 1367 , $1303,1208,1108,1031,908,732,699 \mathrm{~cm}^{-1}$.

\section{Major Diastereomer}

${ }^{1} \mathrm{H} \mathrm{NMR}\left(\mathrm{CDCl}_{3}, 500 \mathrm{MHz}\right): \delta=7.31(\mathrm{t}, J=7.5 \mathrm{~Hz}, 2 \mathrm{H}), 7.22(\mathrm{dd}, J=6.6$, $1.6 \mathrm{~Hz}, 2 \mathrm{H}$ ), $7.20(\mathrm{tt}, J=6.7,1.3 \mathrm{~Hz}, 1 \mathrm{H}), 5.76$ (ddd, $J=15.4,6.7,0.7$ $\mathrm{Hz}, 1 \mathrm{H}$ ), 5.28 (ddd, $J=15.4,9.5,1.4 \mathrm{~Hz}, 1 \mathrm{H}$ ), 3.65 (dd, $J=10.5,5.0 \mathrm{~Hz}$, $1 \mathrm{H}), 3.51$ (pent, $J=6.9 \mathrm{~Hz}, 1 \mathrm{H}$ ), $3.41(\mathrm{dd}, J=10.5,9.0 \mathrm{~Hz}, 1 \mathrm{H}), 2.00$ (tdd, $J=9.6,6.9,5.3 \mathrm{~Hz}, 1 \mathrm{H}$ ), 1.67 (octet, $J=6.7 \mathrm{~Hz}, 1 \mathrm{H}), 1.38(\mathrm{~d}, J=7.0$ $\mathrm{Hz}, 3 \mathrm{H}), 0.93(\mathrm{~d}, J=6.8 \mathrm{~Hz}, 3 \mathrm{H}), 0.89(\mathrm{~d}, J=6.8 \mathrm{~Hz}, 3 \mathrm{H})$.

${ }^{13} \mathrm{C}$ NMR $\left(\mathrm{CDCl}_{3}, 126 \mathrm{MHz}\right): \delta=146.01,139.51,128.45,128.05$, 127.04, 126.06, 64.15, 52.46, 42.47, 28.89, 21.54, 20.86, 19.65.

HRMS (ES+): $m / z$ [241.1568] $]^{+}$calcd for $\mathrm{C}_{15} \mathrm{H}_{22} \mathrm{ONa}^{+}[\mathrm{M}+\mathrm{Na}]^{+}$; found: 241.1558 .

\section{2-(tert-Butyl)-5-phenylhex-3-en-1-ol (51b)}

Prepared according to the general procedure for $\mathrm{SmI}_{2}\left(\mathrm{H}_{2} \mathrm{O}\right)_{n}$ reductions using 50b (0.075 g, $0.212 \mathrm{mmol})$. Purification by flash chromatography on silica gel gave $\mathbf{5 1 b}(0.027 \mathrm{~g}, 73 \%)$ as a colorless oil $($ d.r. = 80:20); $R_{f}=0.33$ ( $4: 1$ hexanes:EtOAc).

IR (ATR): 3436, 3055, 2963, 2873, 1599, 1597, 1512, 1441, 1365, 1255 , $1108,1032,909,732 \mathrm{~cm}^{-1}$.

\section{Major Diastereomer}

${ }^{1} \mathrm{H} \mathrm{NMR}\left(\mathrm{CDCl}_{3}, 500 \mathrm{MHz}\right): \delta=7.30(\mathrm{t}, J=7.65 \mathrm{~Hz}, 2 \mathrm{H}), 7.23-7.17(\mathrm{~m}$, $3 \mathrm{H}$ ), 5.78 (ddd, $J=15.3,6.8,0.6 \mathrm{~Hz}, 1 \mathrm{H}$ ), 5.36 (ddd, $J=15.3,9.9,1.4$ $\mathrm{Hz}, 1 \mathrm{H}), 3.74$ (dd, $J=10.3,3.8 \mathrm{~Hz}, 1 \mathrm{H}$ ), 3.53 (pent, $J=6.8 \mathrm{~Hz}, 1 \mathrm{H}$ ), $3.36(\mathrm{t}, J=10.3 \mathrm{~Hz}, 1 \mathrm{H}), 1.95(\mathrm{td}, J=10.3,4.0 \mathrm{~Hz}, 1 \mathrm{H}), 1.38(\mathrm{~d}, J=7.0$ $\mathrm{Hz}, 3 \mathrm{H}), 0.91$ (s, $9 \mathrm{H})$.

${ }^{13} \mathrm{C}$ NMR $\left(\mathrm{CDCl}_{3}, 126 \mathrm{MHz}\right): \delta=145.99,140.50,128.51,127.52$, $127.01,126.12,61.84,56.49,42.55,32.07,28.08,21.54$.

HRMS (ES+): $m / z$ [255.1725] $]^{+}$calcd for $\mathrm{C}_{16} \mathrm{H}_{24} \mathrm{ONa}^{+}[\mathrm{M}+\mathrm{Na}]^{+}$; found: 255.1725 .

\section{2,5-Diphenylhex-3-en-1-ol (51c)}

Prepared according to the general procedure for $\mathrm{SmI}_{2}\left(\mathrm{H}_{2} \mathrm{O}\right)_{n}$ reductions using 50c $(0.066 \mathrm{~g}, 0.176 \mathrm{mmol})$. Purification by flash chromatography on silica gel gave $51 \mathrm{c}(0.012 \mathrm{~g}, 25 \%)$ as a colorless oil (d.r. $=$ 73:27); $R_{f}=0.28$ (4:1 hexanes:EtOAc).

IR (ATR): 3427, 3026, 2924, 1601, 1492, 1451, 1271, 1031, $758 \mathrm{~cm}^{-1}$.

\section{Major Diastereomer}

${ }^{1} \mathrm{H} \mathrm{NMR}\left(\mathrm{CDCl}_{3}, 500 \mathrm{MHz}\right): \delta=7.34(\mathrm{t}, J=7.6 \mathrm{~Hz}, 2 \mathrm{H}), 7.30(\mathrm{t}, J=7.6$ $\mathrm{Hz}, 2 \mathrm{H}), 7.26-7.17$ (m, $6 \mathrm{H}), 5.82$ (ddd, $J=15.6,6.7,0.88 \mathrm{~Hz}, 1 \mathrm{H}), 5.66$ (ddd, $J=15.4,8.0,1.3 \mathrm{~Hz}, 1 \mathrm{H}$ ), $3.87(\mathrm{~m}, 1 \mathrm{H}$ ), 3.77 (pent, $J=8.15 \mathrm{~Hz}, 2$ H), $3.51(\mathrm{q}, J=7.5 \mathrm{~Hz}, 1 \mathrm{H}) .1 .36(\mathrm{~d}, J=7.1 \mathrm{~Hz}, 3 \mathrm{H})$.

${ }^{13} \mathrm{C}$ NMR $\left(\mathrm{CDCl}_{3}, 126 \mathrm{MHz}\right): \delta=138.13,128.73,128.48,127.90$, $127.12,126.83,126.15,66.51,51.46,42.38,21.40$.

HRMS (ES+): $m / z$ [275.1412 $]^{+}$calcd for $\mathrm{C}_{18} \mathrm{H}_{20} \mathrm{ONa}^{+}[\mathrm{M}+\mathrm{Na}]^{+}$; found: 275.1412 .

\section{2-Benzyl-5-phenylhex-3-en-1-ol (51d)}

Prepared according to the general procedure for $\mathrm{SmI}_{2}\left(\mathrm{H}_{2} \mathrm{O}\right)_{n}$ reductions using 50d $(0.050 \mathrm{~g}, 0.147 \mathrm{mmol})$. Purification by flash chromatography on silica gel gave $\mathbf{5 1 d}(0.030 \mathrm{~g}, 82 \%)$ as a colorless oil (d.r. = 81:19); $R_{f}=0.37$ ( $4: 1$ hexanes:EtOAc).

IR (ATR): 3352, 3056, 3026, 2964, 2925, 2869, 1600, 1580, 1493, 1452 , $1424,1303,1208,1108,1031,973,840,800,700 \mathrm{~cm}^{-1}$.

\section{Major Diastereomer}

${ }^{1} \mathrm{H} \mathrm{NMR}\left(\mathrm{CDCl}_{3}, 500 \mathrm{MHz}\right): \delta=7.29(\mathrm{t}, J=7.1 \mathrm{~Hz}, 2 \mathrm{H}), 7.24(\mathrm{t}, J=7.7$ $\mathrm{Hz}, 2 \mathrm{H}), 7.21(\mathrm{tt}, J=6.2,2.1 \mathrm{~Hz}, 1 \mathrm{H}), 7.19-7.08(\mathrm{~m}, 3 \mathrm{H}), 7.03(\mathrm{~d}, J=$ $7.3 \mathrm{~Hz}, 2 \mathrm{H}$ ), 5.64 (ddd, $J=15.5,6.4,0.8 \mathrm{~Hz}, 1 \mathrm{H}$ ), 5.31 (ddd, $J=15.4$, 8.4, 1.4 Hz, $1 \mathrm{H}$ ), 3.61 (dd, $J=10.5,4.9 \mathrm{~Hz}, 1 \mathrm{H}$ ), 3.48 (dd, $J=10.5,7.5$ $\mathrm{Hz}, 1 \mathrm{H}$ ), 3.42 (pent, $J=6.5 \mathrm{~Hz}, 1 \mathrm{H}), 2.80$ (dd, $J=13.1,6.0 \mathrm{~Hz}, 1 \mathrm{H}$ ), $2.60(\mathrm{dd}, J=13.2,8.4 \mathrm{~Hz}, 1 \mathrm{H}), 2.54(\mathrm{dtd}, J=13.8,7.8,5.0 \mathrm{~Hz}, 1 \mathrm{H}), 1.28$ (d, $J=7.1 \mathrm{~Hz}, 3 \mathrm{H})$.

${ }^{13} \mathrm{C}$ NMR $\left(\mathrm{CDCl}_{3}, 126 \mathrm{MHz}\right): \delta=145.83,139.81,138.48,129.25$, $129.00,128.36,128.23,127.09,126.01,125.92,65.44,47.45,42.14$, 37.88, 21.31.

HRMS (ES+): $m / z$ [289.1568] $]^{+}$calcd for $\mathrm{C}_{19} \mathrm{H}_{22} \mathrm{ONa}^{+}[\mathrm{M}+\mathrm{Na}]^{+}$; found: 289.1563 .

\section{(S)-4-Benzyl-3-\{(S)-2-benzyl-4-[(tert-butyldimethylsilyl)oxy $]$ butanoyl\}oxazolidin-2-one (53)}

To a Schlenk flask containing oxazolidinone $\mathbf{5 2}^{26}$ (1.56 g, $\left.4.14 \mathrm{mmol}\right)$ in THF (20.73 mL) at $-78{ }^{\circ} \mathrm{C}$ was added KHMDS ( $1 \mathrm{M}$ in THF, $9.94 \mathrm{~mL}$, $9.94 \mathrm{mmol}$ ) dropwise. The reaction mixture was stirred at $-78{ }^{\circ} \mathrm{C}$ for $1 \mathrm{~h}$ before freshly distilled benzyl bromide $(1.22 \mathrm{~mL}, 9.94 \mathrm{mmol})$ was added dropwise. The mixture was stirred for $12 \mathrm{~h}$ at $-78{ }^{\circ} \mathrm{C}$ before quenching with aq $\mathrm{NH}_{4} \mathrm{Cl}(40 \mathrm{~mL})$ and extracting with EtOAc $(3 \times 20$ 
$\mathrm{mL})$. The combined organic extracts were dried $\left(\mathrm{MgSO}_{4}\right)$, and concentrated in vacuo. Purification by flash chromatography on silica gel gave 53 ( $1.01 \mathrm{~g}, 62 \%)$ as a colorless oil; $R_{f}=0.51$ (4:1 hexanes:EtOAc). IR (ATR): 3087, 3064, 3028, 2953, 2927, 2855, 1778, 1697, 1603, 1496, $1384,1348,1248,1205,1098,1029,834,775,732,699 \mathrm{~cm}^{-1}$.

${ }^{1} \mathrm{H} \mathrm{NMR}\left(\mathrm{CDCl}_{3}, 500 \mathrm{MHz}\right): \delta=7.28(\mathrm{t}, J=7.4 \mathrm{~Hz}, 4 \mathrm{H}), 7.25(\mathrm{~d}, J=7.0$ $\mathrm{Hz}, 2 \mathrm{H}$ ), 7.19 (tt, J= 5.9, $2.0 \mathrm{~Hz}, 2 \mathrm{H}$ ), 7.07 (d, J = 6.8 Hz, $2 \mathrm{H}$ ), 4.61 (ddt, $J=9.7,7.8,3.1 \mathrm{~Hz}, 1 \mathrm{H}$ ), 4.35 (ddd, $J=7.7,4.6,4.4 \mathrm{~Hz}, 1 \mathrm{H}$ ), 4.09 (ddd, $J=8.6,7.9,0.8 \mathrm{~Hz}, 1 \mathrm{H}), 4.04$, (dd, $J=9.0,2.9 \mathrm{~Hz}, 1 \mathrm{H}), 3.65$ (dd, $J=5.9$, $1.2 \mathrm{~Hz}, 1 \mathrm{H}), 3.64(\mathrm{~d}, J=5.9 \mathrm{~Hz}, 1 \mathrm{H}), 3.07(\mathrm{dd}, J=13.3,7.8 \mathrm{~Hz}, 1 \mathrm{H})$, $3.02(\mathrm{dd}, J=13.5,3.5 \mathrm{~Hz}, 1 \mathrm{H}), 2.80(\mathrm{dd}, J=13.3,7.5 \mathrm{~Hz}, 1 \mathrm{H}), 2.36$ (dd, $J=13.5,9.7 \mathrm{~Hz}, 1 \mathrm{H}), 2.05$ (ddt, $J=13.6,9.1,6.5 \mathrm{~Hz}, 1 \mathrm{H}), 1.71$ (dtd, $J=$ 13.7, 5.8, $4.3 \mathrm{~Hz} 1 \mathrm{H}), 0.85$ (s, $9 \mathrm{H}),-0.01$ (s, $3 \mathrm{H}),-0.02$ (s, $3 \mathrm{H}$ ).

${ }^{13} \mathrm{C}$ NMR $\left(\mathrm{CDCl}_{3}, 126 \mathrm{MHz}\right): \delta=175.83,152.99,138.79,135.42$, $130.12,129.44,129.34,128.87,128.56,128.47,128.31,127.18$, $126.42,65.66,61.41,55.12,41.71,39.15,37.67,34.55,25.93,25.85$, $18.27,-5.49,-5.50$.

HRMS (ES+): $m / z[490.2390]^{+}$calcd for $\mathrm{C}_{27} \mathrm{H}_{37} \mathrm{NO}_{4} \mathrm{SiNa}^{+}[\mathrm{M}+\mathrm{Na}]^{+}$; found: 490.2386 .

\section{(S)-2-Benzyl-4-[(tert-butyldimethylsilyl)oxy]butanal (54)}

To a Schlenk flask containing DCM (100 mL) and 53 (1.014 g, 2.17 $\mathrm{mmol})$ at $-78^{\circ} \mathrm{C}$ was added DIBAL-H $(1.15 \mathrm{~mL}, 6.50 \mathrm{mmol})$ dropwise. The reaction mixture was stirred at $-78{ }^{\circ} \mathrm{C}$ for $4 \mathrm{~h}$ before being warmed to r.t. and quenched with 2 M Rochelle's salt $(100 \mathrm{~mL})$ and stirred for $3 \mathrm{~h}$. The aqueous layer was extracted with DCM $(3 \times 50$ $\mathrm{mL})$. The combined organic extracts were washed with brine $(50 \mathrm{~mL})$, dried $\left(\mathrm{MgSO}_{4}\right)$, and concentrated in vacuo. Purification by flash chromatography on silica gel gave $54(0.358 \mathrm{~g}, 56 \%)$ as a colorless oil; $R_{f}=$ 0.74 (4:1 hexanes:EtOAc).

IR (ATR): 3087, 3064, 3028, 2952, 2927, 2855, 2737, 2713, 1724, 1603 , 1496, 1471, 1388, 1252, 1098, 1029, 987, 833, 809, 774, 730, $698 \mathrm{~cm}^{-1}$.

${ }^{1} \mathrm{H} \mathrm{NMR}\left(\mathrm{CDCl}_{3}, 500 \mathrm{MHz}\right): \delta=\delta 9.71(\mathrm{~d}, J=2.1 \mathrm{~Hz}, 1 \mathrm{H}), 7.29(\mathrm{t}, J=7.6$ $\mathrm{Hz}, 2 \mathrm{H}), 7.20$ (tt, $J=6.7,1.3 \mathrm{~Hz}, 1 \mathrm{H}), 7.17(\mathrm{~d}, J=6.7 \mathrm{~Hz}, 2 \mathrm{H}), 3.67$ (ddd, $J=10.3,6.9,5.1 \mathrm{~Hz}, 1 \mathrm{H}$ ), 3.62 (ddd, $J=10.3,6.7,5.2 \mathrm{~Hz}, 1 \mathrm{H}$ ), $3.04(\mathrm{dd}, J=13.4,6.3 \mathrm{~Hz}, 1 \mathrm{H}), 2.77(\mathrm{dddt}, J=12.0,7.6,4.1,2.0 \mathrm{~Hz}, 1 \mathrm{H})$, $2.71(\mathrm{dd}, J=13.4,7.7 \mathrm{~Hz}, 1 \mathrm{H}), 1.89$ (dddd, $J=14.5,7.9,6.7,5.1 \mathrm{~Hz}, 1 \mathrm{H}$ ) 1.72 (dddd, $J=14.3,6.8,5.2,4.4 \mathrm{~Hz}, 1 \mathrm{H}), 0.87$ (s, $9 \mathrm{H}), 0.03$ (s, $3 \mathrm{H}$ ), $0.02(\mathrm{~s}, 3 \mathrm{H})$.

${ }^{13} \mathrm{C}$ NMR $\left(\mathrm{CDCl}_{3}, 126 \mathrm{MHz}\right): \delta=204.15,138.87,128.99,128.50$, $126.35,60.48,50.68,34.69,31.75,25.86,18.22,-5.51$.

HRMS (ES+): $m / z$ [315.1756] ${ }^{+}$calcd for $\mathrm{C}_{17} \mathrm{H}_{28} \mathrm{O}_{2} \mathrm{SiNa}^{+}[\mathrm{M}+\mathrm{Na}]^{+}$; found: 315.1757 .

\section{(5S,E)-5-Benzyl-7-[(tert-butyldimethylsilyl)oxy]-2-phenylhept-2- en-4-ol (55)}

To a Schlenk flask containing $\mathrm{Et}_{2} \mathrm{O}(12.0 \mathrm{~mL})$ and $t$-BuLi $(1.7 \mathrm{M}, 2.82$ $\mathrm{mL}, 4.8 \mathrm{mmol})$ at $-78{ }^{\circ} \mathrm{C}$ was added vinyl iodide $27^{30}(0.190 \mathrm{~g}, 0.78$ $\mathrm{mmol}$ ) dropwise. The solution was stirred for $5 \mathrm{~min}$ at $-78{ }^{\circ} \mathrm{C}, \mathbf{5 4}$ $(0.585 \mathrm{~g}, 2.4 \mathrm{mmol})$ was then added dropwise, and the reaction was stirred for $1 \mathrm{~h}$ at $-78{ }^{\circ} \mathrm{C}$. The reaction was quenched with aq $\mathrm{NH}_{4} \mathrm{Cl}$ $(30 \mathrm{~mL})$, and extracted with EtOAc $(3 \times 20 \mathrm{~mL})$. The combined organic extracts were dried $\left(\mathrm{MgSO}_{4}\right)$, and concentrated in vacuo. Purification by flash chromatography on silica gel gave $\mathbf{5 5}(0.394 \mathrm{~g}, 93 \%)$ as a colorless oil (d.r. $=62: 38) ; R_{f}=0.44$ (4:1 hexanes:EtOAc).

IR (ATR): 3396, 3083, 3061, 3021, 2927, 2856, 1601, 1494, 1471, 1445 , $1386,1254,1084,1005,908,833,775,757,730,696,664 \mathrm{~cm}^{-1}$.
${ }^{1} \mathrm{H} \mathrm{NMR}\left(\mathrm{CDCl}_{3}, 500 \mathrm{MHz}\right): \delta=7.42(\mathrm{~d}, J=7.5 \mathrm{~Hz}, 2 \mathrm{H}), 7.40(\mathrm{~d} J=8.0$ $\mathrm{Hz}, 2 \mathrm{H}), 7.33(\mathrm{t}, J=7.3 \mathrm{~Hz}, 2 \mathrm{H}), 7.32-7.23(\mathrm{~m}, 10 \mathrm{H}), 7.19(\mathrm{~d}, J=8.1$ $\mathrm{Hz}, 2 \mathrm{H}), 7.17(\mathrm{t}, J=8.2 \mathrm{~Hz}, 2 \mathrm{H}), 5.95(\mathrm{dq}, J=8.8,1.3 \mathrm{~Hz}, 1 \mathrm{H}), 5.89(\mathrm{dq}$, $J=8.7,1.4 \mathrm{~Hz}, 1 \mathrm{H}), 4.61(\mathrm{dt}, J=8.8,4.4 \mathrm{~Hz}, 1 \mathrm{H}), 4.42(\mathrm{dt}, J=8.7,5.4$ $\mathrm{Hz}, 1 \mathrm{H}), 3.80-3.73(\mathrm{~m}, 2 \mathrm{H}), 3.62-3.56(\mathrm{~m}, 2 \mathrm{H}), 3.55(\mathrm{~d}, J=5.2 \mathrm{~Hz}$, $\mathrm{OH}), 3.49(\mathrm{~d}, J=5.3 \mathrm{~Hz}, \mathrm{OH}), 2.89(\mathrm{dd}, J=13.7,6.0 \mathrm{~Hz}, 1 \mathrm{H}), 2.83(\mathrm{dd}$, $J=13.9,5.5 \mathrm{~Hz}, 1 \mathrm{H}), 2.54$ (dd, $J=13.9,9.3 \mathrm{~Hz}, 1 \mathrm{H}), 2.51$ (dd, $J=13.7$, $9.3 \mathrm{~Hz}, 1 \mathrm{H}$ ), 2.21 (octet, $J=4.7 \mathrm{~Hz}, 1 \mathrm{H}$ ), $2.08(\mathrm{~d}, J=1.3 \mathrm{~Hz}, 3 \mathrm{H}), 2.04$ (m, $1 \mathrm{H}), 2.01$ (d, $J=1.3 \mathrm{~Hz}, 3 \mathrm{H}), 1.80(\mathrm{~m}, 1 \mathrm{H}), 1.75$ (dtd, $J=15.3,7.9$, $4.4 \mathrm{~Hz}, 1 \mathrm{H}), 1.66(\mathrm{~m}, 1 \mathrm{H}), 1.55$ (ddt, $J=9.0,6.5,4.3 \mathrm{~Hz}, 1 \mathrm{H}), 0.91(\mathrm{~s}, 9$ H), $0.90(\mathrm{~s}, 9 \mathrm{H}), 0.09(\mathrm{~s}, 3 \mathrm{H}), 0.08(\mathrm{~s}, 3 \mathrm{H}), 0.07(\mathrm{~s}, 3 \mathrm{H}), 0.06(\mathrm{~s}, 3 \mathrm{H})$.

${ }^{13} \mathrm{C}$ NMR $\left(\mathrm{CDCl}_{3}, 126 \mathrm{MHz}\right): \delta=143.42,143.35,140.80,140.78$, $137.38,136.93,130.46,129.13,128.64,128.33,128.28,128.17$, 128.13, 127.09, 127.01, 125.89, 125.87, 125.84, 70.60, 70.53, 62.15, $61.21,45.27,45.24,36.97,36.87,32.40,31.98,25.90,25.89,18.25$, $16.59,16.41,-5.43,-5.46,-5.48$.

HRMS (ES+): $m / z[433.2539]^{+}$calcd for $\mathrm{C}_{26} \mathrm{H}_{38} \mathrm{O}_{2} \mathrm{SiNa}^{+}[\mathrm{M}+\mathrm{Na}]^{+}$; found: 433.2544 .

(5S,E)-5-Benzyl-7-hydroxy-2-phenylhept-2-en-4-yl Benzoate (56) Prepared according to the general benzoylation procedure using $\mathbf{5 5}$ $(0.394 \mathrm{~g}, 0.961 \mathrm{mmol})$. The crude product mixture was placed into a Teflon reaction vessel containing THF $(10 \mathrm{~mL})$, cooled to $0{ }^{\circ} \mathrm{C}$, and treated with HF.pyr (70\% HF, $0.400 \mathrm{~mL}, 12.064 \mathrm{mmol}$ ) and left to sit for $18 \mathrm{~h}$ at $4{ }^{\circ} \mathrm{C}$ without stirring. The reaction was quenched with aq $\mathrm{NaHCO}_{3}(15 \mathrm{~mL})$ and extracted with EtOAc $(3 \times 10 \mathrm{~mL})$. The combined organic extracts were dried $\left(\mathrm{MgSO}_{4}\right)$, and concentrated in vacuo. Purification by flash chromatography over silica gel gave $\mathbf{5 6}(0.312 \mathrm{~g}, 81 \%$ over two steps) as a colorless oil; $R_{f}=0.63$ (1:1 hexanes:EtOAc).

IR (ATR): 3411, 3084, 3061, 3026, 2931, 2880, 1712, 1600, 1583, 1494, 1450, 1381, 1314, 1267, 1175, 1111, 1068, 1025, 907, 757, 730, 710, $696 \mathrm{~cm}^{-1}$.

${ }^{1} \mathrm{H} \mathrm{NMR}\left(\mathrm{CDCl}_{3}, 500 \mathrm{MHz}\right): \delta=8.04(\mathrm{~d}, J=8.0 \mathrm{~Hz}, 4 \mathrm{H}), 7.57(\mathrm{tt}, J=7.5$, $1.3 \mathrm{~Hz}, 1 \mathrm{H}), 7.56$ (tt, $J=7.4,1.4 \mathrm{~Hz}, 1 \mathrm{H}), 7.45$ (t, J = 7.7 Hz, $4 \mathrm{H}), 7.39$ $(\mathrm{t}, J=7.0 \mathrm{~Hz}, 4 \mathrm{H}), 7.35-7.27(\mathrm{~m}, 10 \mathrm{H}), 7.24-7.18(\mathrm{~m}, 6 \mathrm{H}), 6.01(\mathrm{dd}$, $J=9.1,4.7 \mathrm{~Hz}, 1 \mathrm{H}), 5.94(\mathrm{dd}, J=9.2,4.3 \mathrm{~Hz}, 1 \mathrm{H}), 5.91(\mathrm{dq}, J=9.2,1.4$ $\mathrm{Hz}, 1 \mathrm{H}), 5.90(\mathrm{dq}, J=9.2,1.4 \mathrm{~Hz}, 1 \mathrm{H}), 3.71(\mathrm{t}, J=6.8 \mathrm{~Hz}, 4 \mathrm{H}), 2.98(\mathrm{dd}$, $J=14.0,5.8 \mathrm{~Hz}, 1 \mathrm{H}), 2.97(\mathrm{dd}, J=13.7,6.3 \mathrm{~Hz}, 1 \mathrm{H}), 2.70$ (dd, $J=14.0$, $8.5 \mathrm{~Hz}, 1 \mathrm{H}$ ), 2.67 (dd, $J=13.7,8.2 \mathrm{~Hz}, 1 \mathrm{H}$ ), 2.45 (dddd, $J=12.6,8.4$, $6.2,4.9 \mathrm{~Hz}, 1 \mathrm{H}$ ), 2.36 (dddd, $J=12.5,8.2,6.2,4.6 \mathrm{~Hz}, 1 \mathrm{H}), 2.19$ (d, $J=$ $1.3 \mathrm{~Hz}, 3 \mathrm{H}), 2.12(\mathrm{~d}, J=1.2 \mathrm{~Hz}, 3 \mathrm{H}), 1.88(\mathrm{dq}, J=13.6,6.8 \mathrm{~Hz}, 1 \mathrm{H})$, $1.87(\mathrm{dq}, J=13.0,6.9 \mathrm{~Hz}, 1 \mathrm{H}), 1.76(\mathrm{dq}, J=13.5,6.5 \mathrm{~Hz}, 1 \mathrm{H}) 1.66(\mathrm{dq}$, $J=13.2,6.6 \mathrm{~Hz}, 1 \mathrm{H})$.

${ }^{13} \mathrm{C}$ NMR $\left(\mathrm{CDCl}_{3}, 126 \mathrm{MHz}\right): \delta=165.87,142.82,142.78,140.52$, $140.25,140.13,132.93,132.92,130.46,129.61,129.59,129.10$, $129.05,128.50,128.49,128.38,128.25,128.23,127.53,127.49$, $126.17,125.97,125.96,124.23,123.86,73.88,73.71,61.17,61.13$, 42.22, 41.48, 37.08, 36.92, 32.94, 32.87, 16.86, 16.69.

HRMS (ES+): $m / z$ [423.1936 $]^{+}$calcd for $\mathrm{C}_{27} \mathrm{H}_{28} \mathrm{O}_{3} \mathrm{Na}^{+}[\mathrm{M}+\mathrm{Na}]^{+}$; found: 423.1933.

\section{(3S,E)-3-Benzyl-6-phenylhept-4-en-1-ol (57)}

Prepared according to the general procedure for $\mathrm{SmI}_{2}\left(\mathrm{H}_{2} \mathrm{O}\right)_{n}$ reductions using 56 (0.312 g, $0.786 \mathrm{mmol})$. Purification by flash chromatography on silica gel gave $\mathbf{5 7}(0.13 \mathrm{~g}, 32 \%)$ as mixture of diastereomers (d.r. $=74: 26), R_{f}=0.28$ (4:1 hexanes:EtOAc).

IR (ATR): 3084, 3056, 2928, 1600, 1583, 1494, 1450, 1381, 1068, 1025 , $907,757,696 \mathrm{~cm}^{-1}$. 


\section{Major Diastereomer}

${ }^{1} \mathrm{H} \mathrm{NMR}\left(\mathrm{CDCl}_{3}, 500 \mathrm{MHz}\right): \delta=7.40(\mathrm{~d}, J=4.5 \mathrm{~Hz}, 2 \mathrm{H}), 7.36-7.23(\mathrm{~m}, 4$ H), 7.23-7.02 (m, $4 \mathrm{H}), 5.50(\mathrm{dd}, J=15.7,6.9 \mathrm{~Hz}, 1 \mathrm{H}), 5.28$ (ddd, $J=$ 15.6, 9.3, $1.5 \mathrm{~Hz}, 1 \mathrm{H}), 3.77-3.57(\mathrm{~m}, 2 \mathrm{H}), 3.40(\mathrm{~m}, 1 \mathrm{H}), 2.74(\mathrm{dd}, J=$ 13.3, $6.4 \mathrm{~Hz}, 1 \mathrm{H}), 2.63(\mathrm{dd}, J=13.5,8.4 \mathrm{~Hz}, 1 \mathrm{H}), 2.48(\mathrm{~m}, 1 \mathrm{H}), 2.24(\mathrm{t}$, $J=6.8 \mathrm{~Hz}, 1 \mathrm{H}), 1.75(\mathrm{dtd}, J=14.0,7.1,3.9 \mathrm{~Hz}, 1 \mathrm{H}), 1.56(\mathrm{~m}, 1 \mathrm{H}), 1.27$ $(\mathrm{d}, J=7.0 \mathrm{~Hz}, 3 \mathrm{H})$.

${ }^{13} \mathrm{C}$ NMR $\left(\mathrm{CDCl}_{3}, 126 \mathrm{MHz}\right): \delta=146.08,140.91,140.34,136.11$, $132.10,129.42,129.40,129.24,128.59,128.34,128.31,128.20$, $128.15,128.11,127.69,127.15,127.13,127.01,126.63,126.38$, $125.95,125.92,125.84,125.83,125.67,65.40,61.45,61.43,61.13$, $42.54,42.46,42.00,41.98,41.74,40.77,37.63,37.48,37.37,36.68$, $32.72,21.29,16.10$.

HRMS (ES+): $m / z$ [303.1725] $]^{+}$calcd for $\mathrm{C}_{20} \mathrm{H}_{24} \mathrm{ONa}^{+}[\mathrm{M}+\mathrm{Na}]^{+}$; found: 303.1725 .

\section{Funding Information}

National Science Foundation (CHE-1760918)

\section{Supporting Information}

Supporting information for this article is available online at https://doi.org/10.1055/s-0039-1690826.

\section{References}

(1) (a) Namy, J. L.; Girard, P.; Kagan, H. B. New J. Chem. 1977, 1, 5. (b) Kagan, H. B. Tetrahedron 2003, 59, 10351.

(2) (a) Nicolaou, K. C.; Ellery, S. P.; Chen, J. S. Angew. Chem. Int. Ed. 2009, 48, 7140. (b) Edmonds, D. J.; Johnston, D.; Procter, D. J. Chem. Rev. 2004, 104, 3371. (c) Molander, G. A.; Harris, C. R. Chem. Rev. 1996, 96, 843. (d) Szostak, M.; Spain, M.; Parmar, D.; Procter, D. J. Chem. Soc. Rev. 2013, 42, 9155. (e) Procter, D. J.; Flowers, R. A. II.; Skrydstrup, T. Organic Synthesis using Samarium Diiodide; RSC Publishing: Cambridge, 2010. (f) Plesniak, M. P.; Huang, H-M.; Procter, D. J. Nat. Rev. Chem. 2017, 1, 0077.

(3) (a) Enemærke, R. J.; Daasbjerg, K.; Skrydstrup, T. Chem. Commun. 1999, 343. (b) Miller, R. S.; Sealy, J. M.; Shabangi, M.; Kuhlman, M. L.; Fuchs, J. R.; Flowers, R. A. II. J. Am. Chem. Soc. 2000, 122, 7718. (c) Dahlén, A.; Hilmersson, G. Eur. J. Inorg. Chem. 2004, 3393. (d) Sadasivam, D. V.; Teprovich, J. A.; Procter, D. J.; Flowers, R. A. II. Org. Lett. 2010, 12, 4140.

(4) (a) Hutton, T. K.; Muir, K. W.; Procter, D. J. Org. Lett. 2003, 5, 4811. (b) Chopade, P. R.; Prasad, E.; Flowers, R. A. J. Am. Chem. Soc. 2004, 126, 44. (c) Teprovich, J. A. Jr.; Balili, M. N.; Pintauer, T.; Flowers, R. A. II. Angew. Chem. Int. Ed. 2007, 46, 8160. (d) Amiel-Levy, M.; Hoz, S. J. Am. Chem. Soc. 2009, 131, 8280.

(5) (a) Curran, D. P.; Hasegawa, E. J. Org. Chem. 1993, 58, 5008. (b) Szostak, M.; Spain, M.; Parmar, D.; Procter, D. J. Chem. Commun. 2012, 48, 330. (c) Szostak, M.; Spain, M.; Procter, D. J. J. Am. Chem. Soc. 2014, 136, 8459.

(6) (a) Yasuko, K.; Tadahiro, K. Chem. Lett. 1993, 22, 1495. (b) Szostak, M.; Spain, M.; Procter, D. J. Nat. Protoc. 2012, 7, 970. (c) Szostak, M.; Spain, M.; Eberhart, A. J.; Procter, D. J. J. Am. Chem. Soc. 2014, 136, 2268. (d) Huang, H-M.; Procter, D. J. J. Am. Chem. Soc. 2016, 138, 7770.

(7) Berndt, M.; Hölemann, A.; Niermann, A.; Bentz, C.; Zimmer, R.; Reissig, H-U. Eur. J. Org. Chem. 2012, 18093.
(8) Prasad, E.; Flowers, R. A. II. J. Am. Chem. Soc. 2005, 127, 1299.

(9) Chopade, P. R.; Prasad, E.; Flowers, R. A. II. J. Am. Chem. Soc. 2004, 126, 44.

(10) (a) Chciuk, T. V.; Anderson, W. R.; Flowers, R. A. II. J. Am. Chem. Soc. 2016, 138, 8738. (b) Chciuk, T. V.; Anderson, W. R.; Flowers, R. A. II. J. Am. Chem. Soc. 2018, 140, 15342. (c) Chciuk, T. V.; Flowers, R. A. II. J. Am. Chem. Soc. 2015, 137, 11526. (d) Kolmar, S. S.; Mayer, J. M. J. Am. Chem. Soc. 2017, 139, 10687.

(11) (a) O'Neil, G. W.; Moser, D. J.; Volz, E. O. Tetrahedron Lett. 2009, 50, 7355. (b) Volz, E. O.; O'Neil, G. W. J. Org. Chem. 2011, 76, 8428.

(12) Schaefer, S. L.; Roberts, C. L.; Volz, E. O.; Grasso, M. R.; O'Neil, G. W. Tetrahedron Lett. 2013, 54, 6125.

(13) Wright, A. M.; O'Neil, G. W. Tetrahedron Lett. 2016, 57, 3441.

(14) Stockdale, T. F.; O’Neil, G. W. Synlett 2017, 28, 2267.

(15) (a) Farran, H.; Hoz, S. Org. Lett. 2008, 10, 4875. (b) Maity, S.; Flowers, R. A. II.; Hoz, S. Chem. Eur. J. 2017, 23, 17070.

(16) Wipf, P.; Lim, S. Angew. Chem. Int. Ed. 1993, 32, 1068.

(17) Mulzer, J.; Mantoulidis, A.; Öhler, E. J. Org. Chem. 2000, 65, 7456.

(18) (a) Cram, D. J.; Kopecky, K. R. J. Am. Chem. Soc. 1959, 81, 2748. (b) Reetz, M. T. Acc. Chem. Res. 1993, 26, 462.

(19) Keck, G. E.; Wager, C. A. Org. Lett. 2000, 2, 2307.

(20) The reaction was also performed using DMPU and $\mathrm{H}_{2} \mathrm{O}$ together and gave the same d.r. $(75: 25)$ as that obtained when using DMPU (Table 1 , entry 1 ) or $\mathrm{H}_{2} \mathrm{O}$ (entry 5 ).

(21) Compound 6 was converted to a 1:1 mixture of diastereomers by oxidation with Dess-Martin periodinane followed by reduction with $\mathrm{NaBH}_{4}$. See reference 13 .

(22) (a) Banik, B. K.; Venkatraman, M. S.; Banik, I.; Basu, M. K. Tetrahedron Lett. 2004, 45, 4737. (b) Banik, B. K.; Banik, I.; Aounallah, N.; Castillo, M. Tetrahedron Lett. 2005, 46, 7065. (c) Williams, D. B. G.; Caddy, J.; Blann, K.; Grove, J. J. C.; Holzapfel, C. W. Synthesis 2009, 2009. (d) Gómez, A. M.; Uriel, C.; Company, M. D.; López, J. C. Eur.J. Org. Chem. 2011, 7116. (e) Powell, J. R.; Dixon, S.; Light, M. E.; Kilburn, J. D. Tetrahedron Lett. 2009, 50, 3564. (f) Ankner, T.; Hilmersson, G. Tetrahedron Lett. 2007, 48, 5707.

(23) Corey, E. J.; Hannon, F. J.; Boaz, N. W. Tetrahedron 1989, 45, 545.

(24) Eleil, E. L.; Pillar, C. J. Am. Chem. Soc. 1955, 77, 3600.

(25) Prasad, E.; Flowers, R. A. II. J. Am. Chem. Soc. 2002, 124, 6357.

(26) Yu, W.; Zhang, Y.; Jin, Z. Org. Lett. 2001, 3, 1447.

(27) Bied, C.; Kagan, H. B. Tetrahedron 1992, 48, 3877.

(28) Hancock, R. D. J. Chem. Educ. 1992, 69, 615.

(29) Bajpai, R.; Yang, F.; Curran, D. P. Tetrahedron Lett. 2007, 48, 7965.

(30) Mousseau, J. J.; Bull, J. A.; Charette, A. B. Angew. Chem. Int. Ed. 2010, 49, 1115.

(31) Hancock, R. D.; Martell, A. E. Chem. Rev. 1989, 89, 1875.

(32) Instead of an $\eta^{3}$-complex, Sm-II could also be considered as the 4-membered chelate $\mathbf{S m}$-II' (Scheme 12).

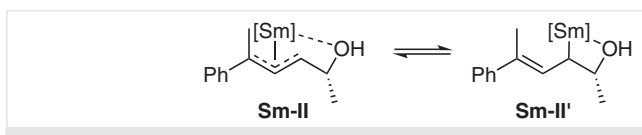

Scheme 12

(33) Kiyotsuka, Y.; Acharya, H. P.; Katayama, Y.; Hyodo, T.; Kobayashi, Y. Org. Lett. 2008, 10, 1719.

(34) Szostak, M.; Spain, M.; Procter, D. J. J. Org. Chem. 2012, 77, 3049.

(35) Spino, C.; Granger, M.-C.; Tremblay, M.-C. Org. Lett. 2002, 4, 4735.

(36) Fuerst, R.; Lentch, C.; Rinner, U. Synthesis 2014, 46, 357. 
Synthesis

T. F. Stockdale et al.

(37) Tanaka-Yanuma, A.; Watanabe, S.; Ogawa, K.; Watanabe, S.; Aoki, N.; Ogura, T.; Usuki, T. Tetrahedron Lett. 2015, 56, 6777.

(38) Fernandes, R. A.; Mulay, S. V.J. Org. Chem. 2010, 75, 7029.

(39) Udagawa, S.; Satoshi, S.; Takemura, T.; Sato, M.; Arai, T.; Nitta, A.; Takumi, A.; Kawai, K.; Iwamura, T.; Okazaki, S.; Takahashi, T.; Kaino, M. Bioorg. Med. Chem. Lett. 2013, 23, 1617.

(40) Sanaboina, C.; Chidara, S.; Jana, S.; Eppakayala, L. Tetrahedron Lett. 2016, 57, 1767.

(41) Angle, S. R.; Bernier, D. S.; Chann, K.; Jones, D. E.; Kim, M.; Neitzel, M. L.; White, S. L. Tetrahedron Lett. 1998, 39, 8195.

(42) Mydock, L. K.; Spilling, C. D.; Demchenko, A. V. C. R. Chim. 2011, 14, 301.

(43) Yadav, J. S.; Nanda, S. Tetrahedron: Asymmetry 2001, 12, 3223. 